Sharif University of Technology
Scientia Iranica
SCIENTIA
IRANICA
http://scientiairanica.sharif.edu

\title{
Pulse extraction of pulse-like ground motions based on particle swarm optimization algorithm
}

\author{
S.R. Hoseini Vaez* and Z. Minaei \\ Department of Civil Engineering, Faculty of Engineering, University of Qom, Qom, Iran.
}

Received 18 November 2017; received in revised form 9 January 2018; accepted 18 June 2018

\author{
KEYWORDS \\ Pulse-like records; \\ Mathematical \\ simulation; \\ Velocity pulse; \\ Particle Swarm \\ Optimization (PSO); \\ Penalty function.
}

\begin{abstract}
Considering the devastating effects of near-fault earthquakes, seismologists and engineers have, qualitatively and quantitatively, represented the strong velocity pulse of near-fault ground motions using models including physical parameters associated with the wave propagation process. In some mathematical models, the derivation of physical parameters is required to fit time history and response spectrum of the simulated record to the actual record through a trial-and-error process, which limits the scope of these models. In the current study, the Particle Swarm Optimization (PSO) algorithm is replaced with the trial-and-error procedure. In this way, an automatic and quantitative process with the minimal judgment of the analyst is prepared to extract a wide range of pulse-like records. Then, the proposed approach is applied to simulate and represent mathematically a set of 91 pulse-like records from the Next Generation Attenuation (NGA) project ground motion library. The obtained results show that a velocity pulse of each pulse-like record could be extracted using the proposed approach; therefore, it can be considered as a powerful tool in pulse parametric studies and the relationship between velocity pulse and structure's response.
\end{abstract}

(C) 2020 Sharif University of Technology. All rights reserved.

\section{Introduction}

In the vicinity of causative faults, when a fault ruptures towards the station with a velocity close to the shear wave velocity, significant seismic energy is often released as a pulse at the beginning of velocity time history. These pulse-like motions have a significant structural damage potential and impose considerable demand on the structure, which cannot be predicted through conventional methods such as elastic-response spectra [1-9].

Because of the destructive effects of such earthquakes, many engineers and seismologists have focused

\footnotetext{
*. Corresponding author. Fax: +98 2532854228

E-mail addresses: hoseinivaez@qom.ac.ir (S.R. Hoseini

Vaez); z.minaei@stu.qom.ac.ir (Z. Minaei)
}

on the quantitative identification and simulation of records containing pulse and studying of structural response to pulse-like motions [10-20]. Baker proposed a criterion for classification of records as pulse-like using wavelet analysis and extracted the largest velocity pulse of these ground motions [21]. To create models that can reliably describe the impulse feature of nearfault motions, Mavroeidis and Papageorgiou [22] and Hoseini Vaez et al. [23] suggested simple mathematical models using unambiguous physical parameters and simulated a set of near-fault records and their corresponding elastic-response spectra. The values of input parameters in the mathematical model are determined by simultaneously fitting its acceleration, velocity, displacement time histories, and the corresponding elastic-response spectra to actual near-fault record based on the trial-and-error process. Recently, Mimoglou et al. [24] determined the parameters of the Mavroeidis and Papageorgiou [22] wavelet by trying 
the pulse parameters in the possible intervals with a specific step. Such a trial-and-error process limits the ability of researchers and engineers to apply these models and examine their effects on research and practical applications because it is actually possible to identify only a limited number of near-field records, and a large number of pulse-like records cannot be extracted in such a time-consuming process.

The application of metaheuristic algorithms, including the Particle Swarm Optimization algorithm (PSO), has been expanded in solving inverse problems and structural optimizations [25-30]. PSO algorithm is a swarm intelligence algorithm based on the social behavior of birds or fish which has been practically used in many research fields due to easy implementation and the algorithm's fast convergence speed [31]. This study focuses on PSO algorithm to simultaneously minimize the difference between the time history and the corresponding elastic-response spectra of the model and those of the actual record. Thus, by applying the optimization algorithm, the largest velocity pulse of the pulse-like ground motions is identified and extracted, and an automatic and quantitative process with the minimal judgment replaces the manual trial-and-error method for processing a wide range of records.

\section{Simulation models of near-fault ground motions}

The model presented by Hoseini Vaez et al. is one of the mathematical models for simulating the strong velocity pulse of near-fault ground motions containing two parts, including both harmonic and polynomial expressions, as shown in Eq. (1) [23]. This model has the capability to simulate various kinds of pulses including unidirectional pulses. Based on Eq. (1), shown in Box I, displacement and acceleration time histories can also be derived in closed form. In Eq. (1), $V(t)$ is the velocity time history, $A$ is the signal amplitude controller, $f_{p}$ is the prevailing frequency, $\nu$ is the phase difference, $\gamma$ represents the oscillatory characteristic of the signal, and $t_{0}$ denotes the time corresponding to the enveloped peak. The model has been fitted using a trial-and-error procedure with some near-fault pulse records in the Next Generation Attenuation (NGA) project library and has simulated the long-period portion of near-fault records with high accuracy. To specify the values of model input parameters, $f_{p}$ has first been determined in a way that pseudo-velocity response spectra of the proposed model and the actual record are almost in an identical period. Phase and time window parameters are determined based on the fitting maximum pulse amplitude and velocity record amplitude in the time window. Then, parameter $A$ has been selected as the pulse amplitude controller parameter by taking into account the amplitude of the actual velocity record and the maximum pseudo-velocity response spectrum. Finally, $\gamma$ parameter, considering its effect on the pulse duration, the number of cycles, and the amplitude of pseudo-velocity response spectrum are specified so that the simulated pulse and the actual record fit desirably [23]. Such a trial-and-error procedure limits the ability of engineers and seismologists to simulate pulse-like near-fault records by using the analytical model. Hence, it is necessary for the trial-and-error process to be replaced by the systematic procedure with the minimal judgment of the analyst.

\section{The optimization algorithm}

\subsection{PSO algorithm}

PSO algorithm, developed by Eberhart and Kennedy, is a randomized algorithm based on population in swarm intelligence and is inspired by the social behavior of birds and fish $[32,33]$. Similar to many optimization algorithms, the PSO method begins with an initial random population as a set of potential solutions. Each element of the population is called a particle. Each particle moves in space based on its own experience and that of other particles. During the optimization process, the best personal experience of each particle and the group's best experience are stored in the algorithm's memory and, by using these data, particle's velocity and position are updated according to Eqs. (2) and (3). Thus, with the repetition of particle movement in space, the knowledge of a good solution is retained by all particles; feasible space is searched, and then the optimal response is found based on personal and collective information.

$$
\begin{aligned}
V_{i}^{k+1}= & w V_{i}^{k}+c_{1} r_{1}\left(\text { Pbest }_{i}^{k}-X_{i}^{k}\right) \\
& +c_{2} r_{2}\left(\text { Gbest }^{k}-X_{i}^{k}\right) \\
X_{i}^{k+1}= & X_{i}^{k}+V_{i}^{k+1}
\end{aligned}
$$

$$
V(t)=\left\{\begin{array}{lc}
A\left(\frac{4 f_{p}}{\gamma}\right)^{4}\left(\left(t-t_{0}\right)^{2}-\left(\frac{\gamma}{4 f_{p}}\right)^{2}\right)^{2} \cos \left(2 \pi f_{p} t+\nu\right), & \left(t_{0}-\frac{\gamma}{4 f_{p}}\right) \leq t \leq\left(t_{0}+\frac{\gamma}{4 f_{p}}\right), \quad \gamma \geq 1 \\
0, & \text { otherwise }
\end{array}\right.
$$


where $k$ is the iteration number, $V_{i}$ is the velocity of the $i$ th particle, $X_{i}$ is the position of the $i$ th particle, Pbest $_{i}$ is the best position of the $i$ th particle, and Gbest is the best particle among all the particles in the population; $c_{1}$ and $c_{2}$ are the learning factors called cognitive parameter and social parameter, respectively; $r_{1}$ and $r_{2}$ are the random numbers with uniform distribution at the interval $[0,1] ; w$ is the local and global exploration controller of the search space, which is called inertia weight and is expressed as the linear reduction function, as in Eq. (4) [31]:

$$
w=w_{\max }-\frac{w_{\max }-w_{\min }}{k_{\max }} k,
$$

where $w_{\max }$ and $w_{\min }$ are the maximum and minimum inertia weights, $k_{\max }$ is the number of algorithm iterations, and $k$ is the current number of iteration, respectively.

\subsection{The penalty function method}

Optimization problems often have constraints that must be satisfied in the optimization process, as defined in the following general form [34]:

$$
\operatorname{Min} f(x) x=\left(x_{1}, \cdots x_{n}\right) \in S \subseteq R^{n},
$$

subject to:

$$
\begin{array}{ll}
g_{p}(x) \leq 0 & p=1,2, \ldots, P, \\
h_{q}(x)=0 & q=1,2, \ldots, Q,
\end{array}
$$

where $f(x), g_{p}(x), h_{q}(x)$, and $x$ are the objective function, the $p$ th inequality constraint, the $q$ th equality constraint, and the $n$-dimensional vector of decision variables, respectively, and $S$ is the feasible region restricted to the lower and upper boundaries of parameters.

Different methods have been developed by researchers to satisfy the constraints in the optimization algorithms, and among these, the penalty function method is the most popular one. From a theoretical and a numerical point of view, the penalty function solves constrained problems by adding value to the objective function proportional to the constraint violation value as in the following:

$$
F(x)=f(x)+\left[\sum_{p=1}^{P} c_{p} G_{p}+\sum_{q=1}^{Q} c_{q} H_{q}\right],
$$

where $F$ is the new objective function to be optimized, and $c_{p}$ and $c_{q}$ are penalty parameters. $G$ and $H$ are the functions of $g$ and $h$ constraints, respectively, to calculate the violation of inequality and equality constraints and are defined as follows:

$$
\begin{aligned}
& G_{p}=\max \left[g_{p}(x), 0\right]^{\beta}, \quad p=1,2, \ldots, P, \\
& H_{q}=\left|h_{q}(x)\right|^{\gamma}, \quad q=1,2, \ldots, Q,
\end{aligned}
$$

where $\gamma$ and $\beta$ are usually 1 or 2 . Therefore, if there is a constraint violation, a positive value is added to the objective function and the algorithm reiterates the previous steps to find the potential solution [35].

\section{The use of an optimization algorithm to simulate the pulse-like motions}

\subsection{Introduction of the proposed approach}

As mentioned earlier, the strong velocity pulse of pulselike ground motions is estimated using the mathematical simulation model through a trial-and-error process and by fitting the displacement, velocity, acceleration time histories, and the corresponding elastic-response spectra obtained through the mathematical model and the actual record. In this study, the trial-and-error process is replaced by a new approach, and to best fit the actual elastic-response spectra and the simulated pulse spectrum, the root-mean-square difference between the two spectra is minimized through the PSO algorithm. In addition, to fit the time histories, the root-meansquare difference between the time history of the actual record and that of the proposed model is considered as the constraint in the optimization process. The penalty function method is used to change the constrained optimization algorithm to a non-constrained algorithm. Thus, the objective function is defined as in Eq. (10) and Figure 1, while gray and black lines display the pseudo-velocity response spectra and velocity time histories of the actual record and simulated pulse, respectively.

$$
F(x)=\operatorname{RMS}\left(S V_{\text {Target }}-S V_{\text {Pulse }}\right)+c H,
$$

where RMS is the Root Mean Square value, $S V_{\text {Target }}$ and $S V_{\text {Pulse }}$ are the pseudo-velocity response spectra of the actual record and the simulated pulse, respectively, $c$ is the penalty parameter, and $H$ indicates the constraint function as follows:

$$
H=\left\{\begin{array}{l}
\operatorname{RMS}\left(V_{\text {Target }}(t)-V_{\text {Pulse }}(t)\right) \\
\text { when the constraint is violated } \\
0, \quad \text { otherwise }
\end{array}\right.
$$

where $V_{\text {Target }}(t)$ and $V_{\text {Pulse }}(t)$ are the velocity time histories of the actual record and the simulated pulse, respectively.

Based on the objective function, optimization variables are the input parameters of the model. According to Eq. (1), five optimization variables are $A, \gamma$, $\nu, f_{p}$, and $t_{0}$, which bound the upper and lower limits of the search space. In addition, considering the characteristics of pulse-like records from the perspective of seismic movements and improving the optimization process performance, constraints were imposed on the generation of a random initial population and particle movement as follows: 


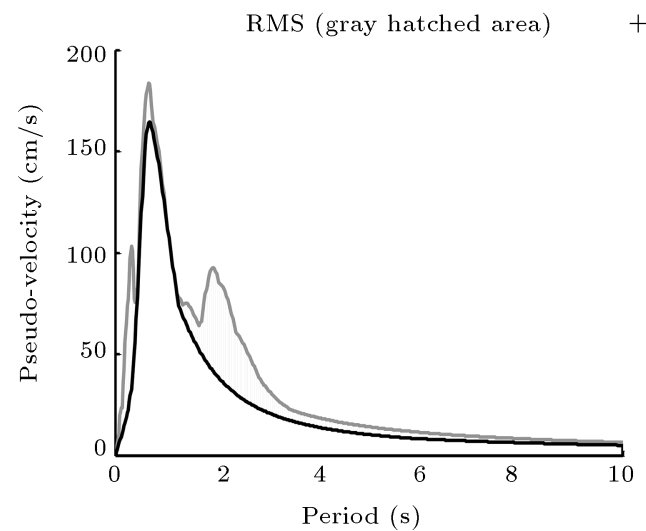

(a)

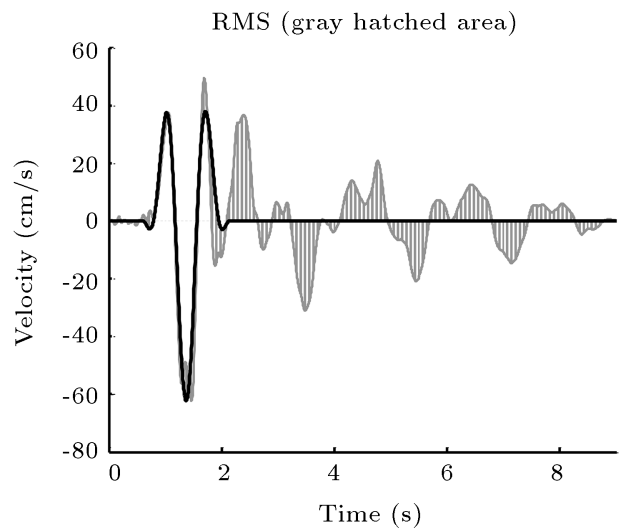

(b)

Figure 1. The RMS difference between: (a) The pseudo-velocity response spectra of the actual record and the simulated pulse (gray hatched area) and (b) the velocity time histories of the actual record and the simulated pulse (gray hatched area).

Table 1. The feasible search space of design variables.

\begin{tabular}{cccccc}
\hline Variable & $\boldsymbol{A}$ & $\boldsymbol{\nu}$ & $\boldsymbol{\gamma}$ & $\boldsymbol{f}_{\boldsymbol{p}}$ & $\boldsymbol{t}_{\mathbf{0}}$ \\
\hline Search space & {$[0.3 \mathrm{PGV}, \mathrm{PGV}]$} & {$[0,2 \pi]$} & {$[4,6]$} & {$[0.6 \mathrm{FA}, \mathrm{FA}]$} & {$\left[t_{P G V}-20 \Delta t, t_{P G V}+20 \Delta t\right]$} \\
\hline
\end{tabular}

1. The Cumulative Squared Velocity (CSV) of ground motions as a seismic parameter affecting the extraction of the strong velocity pulse is defined as in Eq. (12) [36-38]:

$$
\operatorname{CSV}(t)=\int_{t_{1}}^{t_{2}} V^{2}(t) d t
$$

where $t_{1}$ and $t_{2}$ are the starting and ending time points of the velocity time history, respectively. In this study, it is assumed that the energy content of the extracted pulse is smaller than or equal to the actual record. Hence, in the process of generating a random initial population, particles with higher pulse energy content than that of the actual record were not generated. Moreover, particles move in space under this constraint, and if it is not satisfied, particles make a new movement in the search space;

2. In this study, to best fit the actual elastic-response spectra and the simulated pulse spectrum, it is assumed that the simulated pulse spectrum in each period is not larger than the actual record spectrum by more than $5 \%$, and generating a random initial population and particle movement in space should satisfy this condition, too. The flowchart of the proposed approach is shown in Figure 2.

\subsection{Results and discussion}

\subsubsection{Design variables}

By studying a wide variety of recorded ground motions and considering research conducted by Hoseini Vaez et al. [23], the search space of design variables is presented in Table 1. Variable $A$ is a coefficient of Peak Ground Velocity (PGV) as in [0.3PGV, PGV] and $\nu$ in $[0,2 \pi]$. According to Eq. (1), a combination of $\gamma$ and $f_{p}$ parameters specifies the length of time window. Therefore, to determine the time window length, $\gamma$ is in $[4,6]$. Since $f_{p}$ depends on the frequency content of the actual earthquake records and due to the proximity of Fourier amplitude spectrum in two frequencies, interval [0.6FA, FA] is adopted as the prevalent frequency zone, where FA is the maximum Fourier Amplitude. Figure 3 demonstrates the Fourier spectra and the prevalent frequency zone of the 1971 San Fernando earthquake, Pacoima dam (upper left abut). Variable $t_{0}$ is chosen in $\left[t_{P G V}-20 \Delta t, t_{P G V}+20 \Delta t\right]$, where $t_{P G V}$ is the occurrence time of $\mathrm{PGV}$ and $\Delta t$ is the recorded ground motion time step. The defined ranges presented in Table 1 create a five-dimensional feasible space and, during optimization, particles are prevented from moving outside this space.

\subsubsection{Estimating the strong velocity pulse in the mathematical form}

For this study, a wide range of ground motions including 91 NGA records classified as pulse-like by Baker have been investigated [21]. Table 2 presents the specifications of the records. By implementing the proposed approach and the model suggested by Hoseini Vaez et al. [23], the strong velocity pulse of these motions is extracted and represented mathematically.

PSO algorithm parameters are presented in Table 3. The highest velocity pulse in all records is 


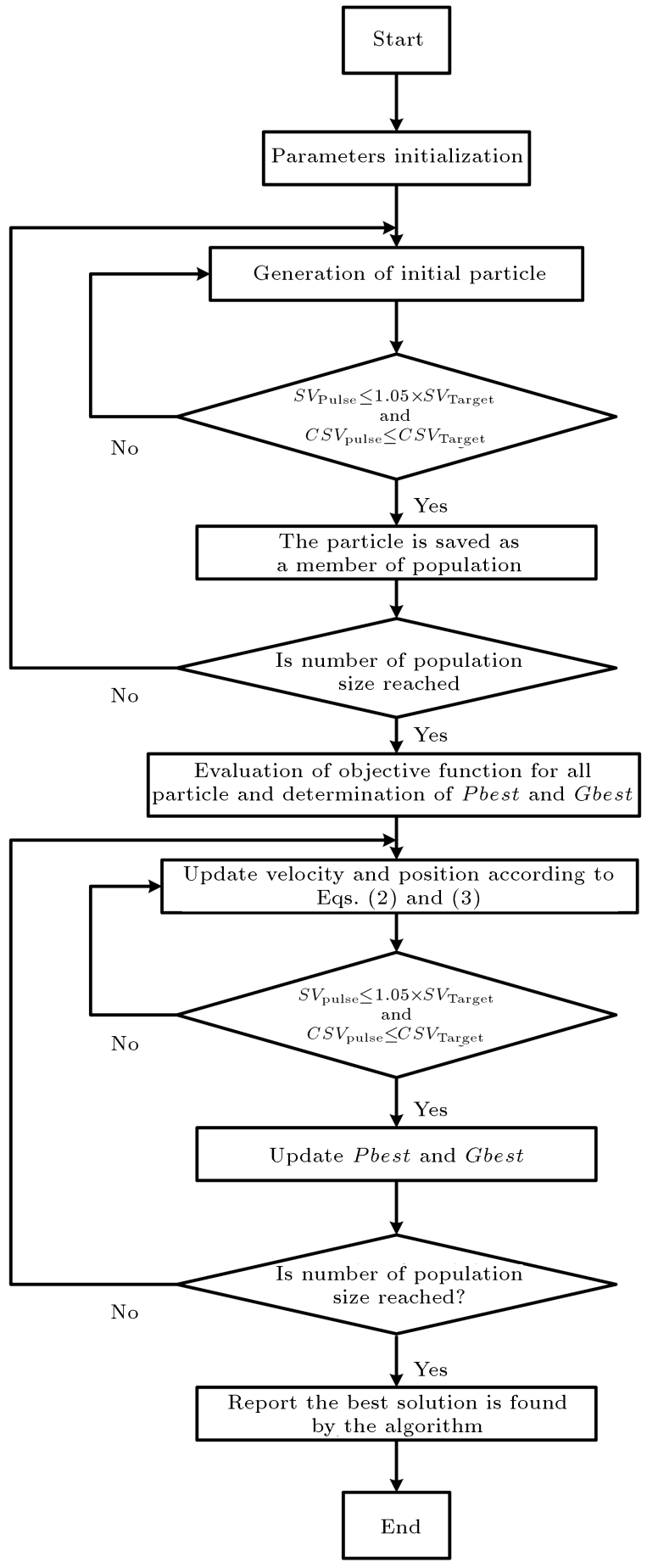

Figure 2. Flowchart demonstration of the proposed approach based on the optimization approach.

extracted using the proposed approach, and the results include parameters $A, \gamma, \nu, f_{p}$, and $t_{0}$, as shown in Table 4. Complete fitting of the simulated pulse and the actual pulse in all pulse-like records is conducted by substituting the parameters of Table 4 in Eq. (1) (see Appendix A).

By using the input parameters in Table 4, the mathematical model fits the displacement, velocity, ac- celeration time histories, and the corresponding elasticresponse spectra of the actual record. In Figure 4, several extracted strong velocity pulses and the original records are shown, and the complete set is also prepared in Appendix A. In addition, the tripartite logarithmic response spectra of a single degree of freedom oscillator with a $5 \%$ damping ratio are illustrated for the extracted pulse and the original record. In these figures, it is observed that the simulated pulse and the actual record are fitted with high quality, and the proposed approach is able to accurately model the long-period portion of the actual record. In addition, the process of convergence to the optimal solution is visible in Figure 5.

\subsubsection{The energy content of the strong velocity pulse} In this section, the energy content of the actual record and the simulated velocity pulse are investigated, according to Eq. (12). Table 5 presents the ratio of $E_{P R}$ and $E_{\text {Pulse }}$ to $E_{\text {Record }}$, where $E_{P R}, E_{\text {Pulse }}$, and $E_{\text {Record }}$ are energy values in the pulse-like region of the actual record, the simulated pulse, and the entire record, respectively. These parameters show that the proposed approach simulates pulse-like records with sufficient accuracy for all records in Table 2. Figure 6 illustrates the energy function of the simulated pulse and the actual record for events, as mentioned in Figures 4 and 5 .

\subsubsection{The prevalent pulse period}

Several methods have been proposed by researchers to determine the velocity pulse period, which is an important parameter for structural engineers. Many researchers consider the zero crossing of the velocity pulse for determining the pulse period, whereas others have used the peak velocity response spectrum. This article defines the pulse period as the inverse of the prevalent frequency of the simulated pulse obtained through the analytical model. This definition is consistent with the physical aspects of the problem and is expressed as in Eq. (13):

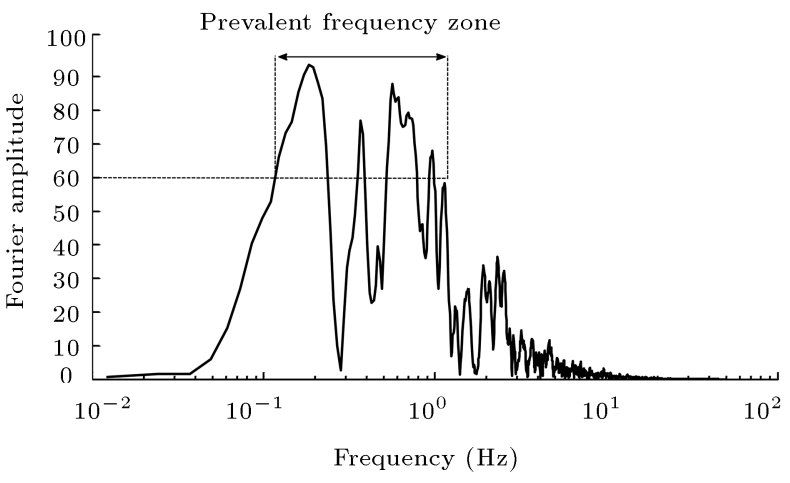

Figure 3. The prevalent frequency zone of the 1971 San Fernando earthquake, Pacoima dam (upper left abut). 
Table 2. Pulse-like ground motions in the NGA database [21].

\begin{tabular}{|c|c|c|c|c|c|c|c|}
\hline \multirow{2}{*}{ No. } & \multirow{2}{*}{ Event } & \multirow{2}{*}{ Year } & \multirow{2}{*}{ Station name } & \multirow{2}{*}{$M_{w}^{\mathrm{a}}$} & \multirow{2}{*}{$\begin{array}{c}\text { PGV } \\
(\mathrm{cm} / \mathrm{s})\end{array}$} & \multicolumn{2}{|c|}{ Distance } \\
\hline & & & & & & Closest $^{\mathrm{b}}(R)$ & Epicentral \\
\hline 1 & San Fernando & 1971 & Pacoima Dam (upper left abut) & 6.6 & 116.5 & 1.8 & 11.9 \\
\hline 2 & Coyote Lake & 1979 & Gilroy array \#6 & 5.7 & 51.5 & 3.1 & 4.4 \\
\hline 3 & Imperial Valley-06 & 1979 & Aeropuerto Mexicali & 6.5 & 44.3 & 0.3 & 2.5 \\
\hline 4 & Imperial Valley-06 & 1979 & Agrarias & 6.5 & 54.4 & 0.7 & 2.6 \\
\hline 5 & Imperial Valley-06 & 1979 & Brawley Airport & 6.5 & 36.1 & 10.4 & 43.2 \\
\hline 6 & Imperial Valley-06 & 1979 & EC County Center FF & 6.5 & 54.5 & 7.3 & 29.1 \\
\hline 7 & Imperial Valley-06 & 1979 & EC Meloland Overpass FF & 6.5 & 115.0 & 0.1 & 19.4 \\
\hline 8 & Imperial Valley-06 & 1979 & El Centro array \#10 & 6.5 & 46.9 & 6.2 & 26.3 \\
\hline 9 & Imperial Valley-06 & 1979 & El Centro array \#11 & 6.5 & 41.1 & 12.5 & 29.4 \\
\hline 10 & Imperial Valley-06 & 1979 & El Centro array \#3 & 6.5 & 41.1 & 12.9 & 28.7 \\
\hline 11 & Imperial Valley-06 & 1979 & El Centro array \#4 & 6.5 & 77.9 & 7.1 & 27.1 \\
\hline 12 & Imperial Valley-06 & 1979 & El Centro array \#5 & 6.5 & 91.5 & 4.0 & 27.8 \\
\hline 13 & Imperial Valley-06 & 1979 & El Centro array \#6 & 6.5 & 111.9 & 1.4 & 27.5 \\
\hline 14 & Imperial Valley-06 & 1979 & El Centro array \#7 & 6.5 & 108.8 & 0.6 & 27.6 \\
\hline 15 & Imperial Valley-06 & 1979 & El Centro array \#8 & 6.5 & 48.6 & 3.9 & 28.1 \\
\hline 16 & Imperial Valley-06 & 1979 & El Centro differential array & 6.5 & 59.6 & 5.1 & 27.2 \\
\hline 17 & Imperial Valley-06 & 1979 & Holtville Post Office & 6.5 & 55.1 & 7.7 & 19.8 \\
\hline 18 & Mammoth Lakes-06 & 1980 & Long Valley Dam (Upr L abut) & 5.9 & 33.1 & - & 14.0 \\
\hline 19 & Irpinia, Italy-01 & 1980 & Sturno & 6.9 & 41.5 & 10.8 & 30.4 \\
\hline 20 & Westmorland & 1981 & Parachute test site & 5.9 & 35.8 & 16.7 & 20.5 \\
\hline 21 & Coalinga-05 & 1983 & Oil City & 5.8 & 41.2 & - & 4.6 \\
\hline 22 & Coalinga-05 & 1983 & Transmitter Hill & 5.8 & 46.1 & - & 6.0 \\
\hline 23 & Coalinga-07 & 1983 & Coalinga-14th \& Elm (old CHP) & 5.2 & 36.1 & - & 9.6 \\
\hline 24 & Morgan Hill & 1984 & Coyote Lake Dam (SW abut) & 6.2 & 62.3 & 0.5 & 24.6 \\
\hline 25 & Morgan Hill & 1984 & Gilroy array \#6 & 6.2 & 35.4 & 9.9 & 36.3 \\
\hline 26 & Taiwan SMART1(40) & 1986 & SMART1 C00 & 6.3 & 31.2 & - & 68.2 \\
\hline 27 & Taiwan SMART1(40) & 1986 & SMART1 M07 & 6.3 & 36.1 & - & 67.2 \\
\hline 28 & N. Palm Springs & 1986 & North Palm Springs & 6.1 & 73.6 & 4.0 & 10.6 \\
\hline 29 & San Salvador & 1986 & Geotech investing center & 5.8 & 62.3 & 6.3 & 7.9 \\
\hline 30 & Whittier Narrows-01 & 1987 & Downey - Co Maint Bldg & 6.0 & 30.4 & 20.8 & 16.0 \\
\hline 31 & Whittier Narrows-01 & 1987 & LB - Orange Ave & 6.0 & 32.9 & 24.5 & 20.7 \\
\hline 32 & Superstition Hills-02 & 1987 & Parachute test site & 6.5 & 106.8 & 1.0 & 16.0 \\
\hline 33 & Loma Prieta & 1989 & Alameda Naval Air Stn Hanger & 6.9 & 32.2 & 71.0 & 90.8 \\
\hline 34 & Loma Prieta & 1989 & Gilroy array \#2 & 6.9 & 45.7 & 11.1 & 29.8 \\
\hline 35 & Loma Prieta & 1989 & Oakland - Outer Harbor Wharf & 6.9 & 49.2 & 74.3 & 94.0 \\
\hline
\end{tabular}

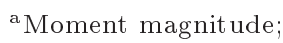

${ }^{\mathrm{b}}$ Closest distance from the recording site to the ruptured area (if available);

${ }^{c}$ Distance from the recording site to the epicenter. 
Table 2. Pulse-like ground motions in the NGA database [21] (continued).

\begin{tabular}{|c|c|c|c|c|c|c|c|}
\hline \multirow{2}{*}{ No. } & \multirow{2}{*}{ Event } & \multirow{2}{*}{ Year } & \multirow{2}{*}{ Station name } & \multirow{2}{*}{$M_{w}^{\mathrm{a}}$} & \multirow{2}{*}{$\begin{array}{c}\text { PGV } \\
(\mathrm{cm} / \mathrm{s})\end{array}$} & \multicolumn{2}{|c|}{ Distance } \\
\hline & & & & & & Closest $^{\mathrm{b}}(R)$ & Epicentral $^{\mathrm{c}}$ \\
\hline 36 & Loma Prieta & 1989 & Saratoga - Aloha Ave & 6.9 & 55.6 & 8.5 & 27.2 \\
\hline 37 & Erzincan, Turkey & 1992 & Erzincan & 6.7 & 95.4 & 4.4 & 9.0 \\
\hline 38 & Cape Mendocino & 1992 & Petrolia & 7.0 & 82.1 & 8.2 & 4.5 \\
\hline 39 & Landers & 1992 & Barstow & 7.3 & 30.4 & 34.9 & 94.8 \\
\hline 40 & Landers & 1992 & Lucerne & 7.3 & 140.3 & 2.2 & 44.0 \\
\hline 41 & Landers & 1992 & Yermo Fire Station & 7.3 & 53.2 & 23.6 & 86.0 \\
\hline 42 & Northridge-01 & 1994 & Jensen Filter Plant & 6.7 & 67.4 & 5.4 & 13.0 \\
\hline 43 & Northridge-01 & 1994 & Jensen Filter Plant Generator & 6.7 & 67.4 & 5.4 & 13.0 \\
\hline 44 & Northridge-01 & 1994 & LA- Wadsworth VA Hospital North & 6.7 & 32.4 & 23.6 & 19.6 \\
\hline 45 & Northridge-01 & 1994 & LA Dam & 6.7 & 77.1 & 5.9 & 11.8 \\
\hline 46 & Northridge-01 & 1994 & Newhall - W Pico Canyon Rd. & 6.7 & 87.8 & 5.5 & 21.6 \\
\hline 47 & Northridge-01 & 1994 & Pacoima Dam (downstr) & 6.7 & 50.4 & 7.0 & 20.4 \\
\hline 48 & Northridge-01 & 1994 & Pacoima Dam (upper left) & 6.7 & 107.1 & 7.0 & 20.4 \\
\hline 49 & Northridge-01 & 1994 & Rinaldi Receiving Sta & 6.7 & 167.2 & 6.5 & 10.9 \\
\hline 50 & Northridge-01 & 1994 & Sylmar - Converter Sta & 6.7 & 130.3 & 5.4 & 13.1 \\
\hline 51 & Northridge-01 & 1994 & Sylmar - Converter Sta East & 6.7 & 116.6 & 5.2 & 13.6 \\
\hline 52 & Northridge-01 & 1994 & Sylmar - Olive View med FF & 6.7 & 122.7 & 5.3 & 16.8 \\
\hline 53 & Kobe, Japan & 1995 & Takarazuka & 6.9 & 72.6 & 0.3 & 38.6 \\
\hline 54 & Kobe, Japan & 1995 & Takatori & 6.9 & 169.6 & 1.5 & 13.1 \\
\hline 55 & Kocaeli, Turkey & 1999 & Gebze & 7.5 & 52.0 & 10.9 & 47.0 \\
\hline 56 & Chi-Chi, Taiwan & 1999 & CHY006 & 7.6 & 64.7 & 9.8 & 40.5 \\
\hline 57 & Chi-Chi, Taiwan & 1999 & CHY035 & 7.6 & 42.0 & 12.7 & 43.9 \\
\hline 58 & Chi-Chi, Taiwan & 1999 & CHY101 & 7.6 & 85.4 & 10.0 & 32.0 \\
\hline 59 & Chi-Chi, Taiwan & 1999 & TAP003 & 7.6 & 33.0 & 102.4 & 151.7 \\
\hline 60 & Chi-Chi, Taiwan & 1999 & TCU029 & 7.6 & 62.3 & 28.1 & 79.2 \\
\hline 61 & Chi-Chi, Taiwan & 1999 & TCU031 & 7.6 & 59.9 & 30.2 & 80.1 \\
\hline 62 & Chi-Chi, Taiwan & 1999 & TCU034 & 7.6 & 42.8 & 35.7 & 87.9 \\
\hline 63 & Chi-Chi, Taiwan & 1999 & TCU036 & 7.6 & 62.4 & 19.8 & 67.8 \\
\hline 64 & Chi-Chi, Taiwan & 1999 & TCU038 & 7.6 & 50.9 & 25.4 & 73.1 \\
\hline 65 & Chi-Chi, Taiwan & 1999 & TCU040 & 7.6 & 53.0 & 22.1 & 69.0 \\
\hline 66 & Chi-Chi, Taiwan & 1999 & TCU042 & 7.6 & 47.3 & 26.3 & 78.4 \\
\hline 67 & Chi-Chi, Taiwan & 1999 & TCU046 & 7.6 & 44.0 & 16.7 & 68.9 \\
\hline 68 & Chi-Chi, Taiwan & 1999 & TCU049 & 7.6 & 44.8 & 3.8 & 38.9 \\
\hline 69 & Chi-Chi, Taiwan & 1999 & TCU053 & 7.6 & 41.9 & 6.0 & 41.2 \\
\hline
\end{tabular}

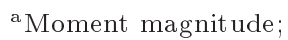

${ }^{\mathrm{b}}$ Closest distance from the recording site to the ruptured area (if available);

${ }^{\mathrm{c}}$ Distance from the recording site to the epicenter. 
Table 2. Pulse-like ground motions in the NGA database [21] (continued).

\begin{tabular}{|c|c|c|c|c|c|c|c|}
\hline \multirow{2}{*}{ No. } & \multirow{2}{*}{ Event } & \multirow{2}{*}{ Year } & \multirow{2}{*}{ Station name } & \multirow{2}{*}{$M_{w}{ }^{\mathrm{a}}$} & \multirow{2}{*}{$\begin{array}{l}\text { PGV } \\
(\mathrm{cm} / \mathrm{s})\end{array}$} & \multicolumn{2}{|c|}{ Distance } \\
\hline & & & & & & Closest $^{\mathrm{b}}(R)$ & Epicentral $^{\mathrm{c}}$ \\
\hline 70 & Chi-Chi, Taiwan & 1999 & TCU054 & 7.6 & 60.9 & 5.3 & 37.6 \\
\hline 71 & Chi-Chi, Taiwan & 1999 & TCU056 & 7.6 & 43.5 & 10.5 & 39.7 \\
\hline 72 & Chi-Chi, Taiwan & 1999 & TCU060 & 7.6 & 33.7 & 8.5 & 45.4 \\
\hline 73 & Chi-Chi, Taiwan & 1999 & TCU065 & 7.6 & 127.7 & 0.6 & 26.7 \\
\hline 74 & Chi-Chi, Taiwan & 1999 & TCU068 & 7.6 & 191.1 & 0.3 & 47.9 \\
\hline 75 & Chi-Chi, Taiwan & 1999 & TCU075 & 7.6 & 88.4 & 0.9 & 20.7 \\
\hline 76 & Chi-Chi, Taiwan & 1999 & TCU076 & 7.6 & 63.7 & 2.8 & 16.0 \\
\hline 77 & Chi-Chi, Taiwan & 1999 & TCU082 & 7.6 & 56.1 & 5.2 & 36.2 \\
\hline 78 & Chi-Chi, Taiwan & 1999 & TCU087 & 7.6 & 53.7 & 7.0 & 55.6 \\
\hline 79 & Chi-Chi, Taiwan & 1999 & TCU098 & 7.6 & 32.7 & 47.7 & 99.7 \\
\hline 80 & Chi-Chi, Taiwan & 1999 & TCU101 & 7.6 & 68.4 & 2.1 & 45.1 \\
\hline 81 & Chi-Chi, Taiwan & 1999 & TCU102 & 7.6 & 106.6 & 1.5 & 45.6 \\
\hline 82 & Chi-Chi, Taiwan & 1999 & TCU103 & 7.6 & 62.2 & 6.1 & 52.4 \\
\hline 83 & Chi-Chi, Taiwan & 1999 & TCU104 & 7.6 & 31.4 & 12.9 & 49.3 \\
\hline 84 & Chi-Chi, Taiwan & 1999 & TCU128 & 7.6 & 78.7 & 13.2 & 63.3 \\
\hline 85 & Chi-Chi, Taiwan & 1999 & TCU136 & 7.6 & 51.8 & 8.3 & 48.8 \\
\hline 86 & Northwest China-03 & 1997 & Jiashi & 6.1 & 37.0 & - & 19.1 \\
\hline 87 & Yountville & 2000 & Napa Fire Station \#3 & 5.0 & 43.0 & - & 9.9 \\
\hline 88 & Chi-Chi, Taiwan-03 & 1999 & CHY024 & 6.2 & 33.1 & 19.7 & 25.5 \\
\hline 89 & Chi-Chi, Taiwan-03 & 1999 & CHY080 & 6.2 & 69.9 & 22.4 & 29.5 \\
\hline 90 & Chi-Chi, Taiwan-03 & 1999 & TCU076 & 6.2 & 59.4 & 14.7 & 20.8 \\
\hline 91 & Chi-Chi, Taiwan-06 & 1999 & CHY101 & 6.3 & 36.3 & 36.0 & 50.0 \\
\hline
\end{tabular}

Table 3. PSO algorithm parameters.

\begin{tabular}{cccccccccc}
\hline Parameter & Population size & $\boldsymbol{k}_{\max }$ & $\boldsymbol{c}_{\mathbf{1}}$ & $\boldsymbol{c}_{\mathbf{2}}$ & $\boldsymbol{w}_{\min }$ & $\boldsymbol{w}_{\max }$ & $\boldsymbol{c}$ & $\boldsymbol{\gamma}$ \\
\hline Value & 50 & 200 & 2 & 2 & 0.8 & 1.4 & 5 & 1 \\
\hline
\end{tabular}

$$
T_{p}=\frac{1}{f_{p}},
$$

where $T_{p}$ is the prevalent pulse period. It should be noted that, in the literature, much attention is devoted to the relationship between the prevalent pulse period and the earthquake magnitude. Figure 7 indicates the prevalent pulse period versus earthquake magnitude based on Eq. (13) and Table 4. In this regard, Eq. (14) is generated from a linear regression analysis of the prevalent pulse period and the earthquake magnitude, very similar to those existing in the literature [21]:

$$
\log \left(T_{p}\right)=-2.3+0.4 M_{w}
$$


Table 4. Input parameters obtained by fitting the pulse-like records in Table 2 to Hoseini Vaez's model.

\begin{tabular}{|c|c|c|c|c|c|c|c|c|}
\hline No. & Event & Year & Station name & $A$ & $\gamma$ & $\nu$ & $f_{p}$ & $t_{0}$ \\
\hline 1 & San Fernando & 1971 & Pacoima Dam (upper left abut) & 94.51 & 4 & 4.55 & 0.751 & 3.09 \\
\hline 2 & Coyote Lake & 1979 & Gilroy array \#6 & 37.35 & 4 & 0.9 & 1.06 & 2.7 \\
\hline 3 & Imperial Valley-06 & 1979 & Aeropuerto Mexicali & 34.93 & 4 & 5.66 & 0.569 & 5.4 \\
\hline 4 & Imperial Valley-06 & 1979 & Agrarias & 38.54 & 4 & 1.82 & 0.485 & 7.74 \\
\hline 5 & Imperial Valley-06 & 1979 & Brawley Airport & 28.79 & 4 & 0 & 0.281 & 7.33 \\
\hline 6 & Imperial Valley-06 & 1979 & EC county Center FF & 41.43 & 4 & 3.9 & 0.25 & 7.48 \\
\hline 7 & Imperial Valley-06 & 1979 & EC meloland Overpass FF & 79.52 & 4.006 & 1.19 & 0.365 & 4.91 \\
\hline 8 & Imperial Valley-06 & 1979 & El Centro array \#10 & 34.55 & 4 & 4.42 & 0.241 & 7.07 \\
\hline 9 & Imperial Valley-06 & 1979 & El Centro array \#11 & 17.12 & 5.102 & 1.15 & 0.152 & 8.69 \\
\hline 10 & Imperial Valley-06 & 1979 & El Centro array \#3 & 26.89 & 4 & 4.32 & 0.218 & 8.36 \\
\hline 11 & Imperial Valley-06 & 1979 & El Centro array \#4 & 66.41 & 4 & 5.68 & 0.236 & 5.29 \\
\hline 12 & Imperial Valley-06 & 1979 & El Centro array \#5 & 78.92 & 4 & 3.28 & 0.273 & 7.41 \\
\hline 13 & Imperial Valley-06 & 1979 & El Centro array \#6 & 94.13 & 4 & 3.41 & 0.267 & 5.8 \\
\hline 14 & Imperial Valley-06 & 1979 & El Centro array \#7 & 73.5 & 4.072 & 2.02 & 0.287 & 6.04 \\
\hline 15 & Imperial Valley-06 & 1979 & El Centro array \#8 & 39.92 & 4.18 & 5.42 & 0.22 & 6.98 \\
\hline 16 & Imperial Valley-06 & 1979 & El Centro differential array & 36.07 & 4 & 5.94 & 0.213 & 6.86 \\
\hline 17 & Imperial Valley-06 & 1979 & Holtville Post Office & 40.03 & 4.003 & 0 & 0.216 & 7.28 \\
\hline 18 & Mammoth lakes-06 & 1980 & Long Valley Dam (Upr L abut) & 24.22 & 4.573 & 2.76 & 1.003 & 5.19 \\
\hline 19 & Irpinia, Italy-01 & 1980 & Sturno & 40.77 & 4.096 & 1.47 & 0.347 & 8.1 \\
\hline 20 & Westmorland & 1981 & Parachute test site & 27.58 & 4 & 2.5 & 0.317 & 11.12 \\
\hline 21 & Coalinga-05 & 1983 & Oil city & 40.61 & 4 & 4.69 & 1.655 & 2.93 \\
\hline 22 & Coalinga-05 & 1983 & Transmitter Hill & 46.06 & 5.363 & 4.04 & 1.325 & 3.05 \\
\hline 23 & Coalinga-07 & 1983 & Coalinga-14th \& Elm (old CHP) & 34.86 & 4 & 0.67 & 2.575 & 2.82 \\
\hline 24 & Morgan Hill & 1984 & Coyote lake Dam (SW abut) & 51.64 & 5.29 & 5.34 & 1.267 & 3.76 \\
\hline 25 & Morgan Hill & 1984 & Gilroy array \#6 & 26.64 & 5.212 & 0.71 & 0.927 & 5.89 \\
\hline 26 & Taiwan SMART1(40) & 1986 & SMART1 C00 & 30.49 & 4.147 & 0.9 & 0.729 & 7.36 \\
\hline 27 & Taiwan SMART1(40) & 1986 & SMART1 M07 & 36.12 & 4 & 0 & 0.731 & 11.71 \\
\hline 28 & N. Palm springs & 1986 & North Palm Springs & 51.82 & 4 & 3.78 & 0.819 & 2.84 \\
\hline 29 & San Salvador & 1986 & Geotech Investig Center & 62.26 & 4 & 4.55 & 1.294 & 1.38 \\
\hline 30 & Whittier Narrows-01 & 1987 & Downey - Co Maint Bldg & 25.42 & 5.002 & 0.96 & 1.222 & 5.53 \\
\hline 31 & Whittier Narrows-01 & 1987 & LB - Orange Ave & 25.75 & 5.16 & 1.96 & 1.226 & 6.09 \\
\hline 32 & Superstition Hills-02 & 1987 & Parachute Test Site & 91.79 & 4 & 2.55 & 0.467 & 12.31 \\
\hline 33 & Loma Prieta & 1989 & Alameda Naval Air Stn Hanger & 22.25 & 4 & 1.55 & 0.464 & 12.29 \\
\hline 34 & Loma Prieta & 1989 & Gilroy array \#2 & 37.29 & 5.211 & 3.48 & 0.654 & 4.02 \\
\hline 35 & Loma Prieta & 1989 & Oakland - Outer Harbor Wharf & 38.61 & 4 & 1.17 & 0.631 & 12.96 \\
\hline 36 & Loma Prieta & 1989 & Saratoga - Aloha Ave & 28.36 & 4 & 4.05 & 0.273 & 6.36 \\
\hline 37 & Erzican, Turkey & 1992 & Erzincan & 74.79 & 4 & 2.47 & 0.458 & 3.45 \\
\hline 38 & Cape Mendocino & 1992 & Petrolia & 45.07 & 4 & 3.87 & 0.404 & 3.16 \\
\hline 39 & Landers & 1992 & Barstow & 19.14 & 4 & 5.65 & 0.14 & 17.66 \\
\hline 40 & Landers & 1992 & Lucerne & 78.13 & 4 & 6.07 & 0.208 & 10.15 \\
\hline 41 & Landers & 1992 & Yermo Fire Station & 37.91 & 4 & 4.86 & 0.148 & 18.21 \\
\hline 42 & Northridge-01 & 1994 & Jensen Filter Plant & 64.46 & 4.547 & 1.69 & 0.341 & 3.32 \\
\hline 43 & Northridge-01 & 1994 & Jensen Filter Plant Generator & 65.31 & 4.365 & 1.79 & 0.336 & 3.32 \\
\hline 44 & Northridge-01 & 1994 & LA - Wadsworth VA Hospital North & 18.11 & 4 & 0.87 & 0.439 & 9.72 \\
\hline 45 & Northridge-01 & 1994 & LA Dam & 51.39 & 4 & 3.38 & 0.698 & 2.9 \\
\hline 46 & Northridge-01 & 1994 & Newhall - W Pico Canyon Rd. & 78.51 & 4 & 3.5 & 0.429 & 5.43 \\
\hline
\end{tabular}

aThese records are extracted and simulated in different parameter ranges than those in Table 1. 
Table 4. Input parameters obtained by fitting the pulse-like records in Table 2 to Hoseini Vaez's model (continued).

\begin{tabular}{|c|c|c|c|c|c|c|c|c|}
\hline No. & Event & Year & Station name & $A$ & $\gamma$ & $\nu$ & $f_{p}$ & $t_{0}$ \\
\hline $47^{\mathrm{a}}$ & Northridge-01 & 1994 & Pacoima Dam (downstr) & 30.86 & 4.827 & 4.78 & 1.984 & 3.50 \\
\hline 48 & Northridge-01 & 1994 & Pacoima Dam (upper left) & 88.72 & 4 & 6.01 & 1.191 & 3.97 \\
\hline 49 & Northridge-01 & 1994 & Rinaldi receiving Sta & 120.96 & 4 & 3.43 & 0.813 & 2.58 \\
\hline 50 & Northridge-01 & 1994 & Sylmar - Converter Sta & 83.11 & 4.32 & 1.38 & 0.349 & 3.6 \\
\hline 51 & Northridge-01 & 1994 & Sylmar - Converter Sta East & 61.68 & 4 & 1.84 & 0.332 & 3.43 \\
\hline 52 & Northridge-01 & 1994 & Sylmar - Olive View med FF & 77.59 & 4 & 5.3 & 0.44 & 3.97 \\
\hline 53 & Kobe, Japan & 1995 & Takarazuka & 61.20 & 4 & 3.88 & 0.724 & 5.36 \\
\hline 54 & Kobe, Japan & 1995 & Takatori & 132.88 & 4 & 3.91 & 0.639 & 6.11 \\
\hline $55^{\mathrm{a}}$ & Kocaeli, Turkey & 1999 & Gebze & 37.80 & 4 & 0.55 & 0.185 & 7.74 \\
\hline 56 & Chi-Chi, Taiwan & 1999 & CHY006 & 46.99 & 4 & 3.7 & 0.43 & 34.52 \\
\hline 57 & Chi-Chi, Taiwan & 1999 & CHY035 & 34.67 & 4.686 & 2.95 & 0.662 & 35.55 \\
\hline 58 & Chi-Chi, Taiwan & 1999 & CHY101 & 58.03 & 4 & 0 & 0.205 & 38.91 \\
\hline 59 & Chi-Chi, Taiwan & 1999 & TAP003 & 27.15 & 5.167 & 2.65 & 0.333 & 46.75 \\
\hline 60 & Chi-Chi, Taiwan & 1999 & TCU029 & 54.78 & 4.309 & 4.92 & 0.18 & 51.19 \\
\hline 61 & Chi-Chi, Taiwan & 1999 & TCU031 & 59.86 & 4.649 & 2.25 & 0.199 & 53.74 \\
\hline 62 & Chi-Chi, Taiwan & 1999 & TCU034 & 22.91 & 5.121 & 6.28 & 0.138 & 47.3 \\
\hline 63 & Chi-Chi, Taiwan & 1999 & TCU036 & 55.85 & 4.042 & 1.95 & 0.176 & 46.73 \\
\hline 64 & Chi-Chi, Taiwan & 1999 & TCU038 & 48.07 & 4 & 2.57 & 0.152 & 47.28 \\
\hline 65 & Chi-Chi, Taiwan & 1999 & TCU040 & 45.23 & 4.67 & 4.96 & 0.183 & 47.81 \\
\hline 66 & Chi-Chi, Taiwan & 1999 & TCU042 & 31.43 & 4 & 0 & 0.134 & 48.15 \\
\hline 67 & Chi-Chi, Taiwan & 1999 & TCU046 & 30.91 & 4.191 & 1.45 & 0.133 & 39.88 \\
\hline 68 & Chi-Chi, Taiwan & 1999 & TCU049 & 34.33 & 4 & 2.07 & 0.099 & 37.59 \\
\hline 69 & Chi-Chi, Taiwan & 1999 & TCU053 & 26.78 & 4 & 3.19 & 0.093 & 36.66 \\
\hline 70 & Chi-Chi, Taiwan & 1999 & TCU054 & 38.67 & 4 & 0.63 & 0.135 & 36.19 \\
\hline 71 & Chi-Chi, Taiwan & 1999 & TCU056 & 23.86 & 4 & 1.26 & 0.11 & 38.47 \\
\hline 72 & Chi-Chi, Taiwan & 1999 & TCU060 & 21.88 & 4.046 & 0.78 & 0.083 & 41 \\
\hline 73 & Chi-Chi, Taiwan & 1999 & TCU065 & 93.45 & 5.49 & 3.02 & 0.207 & 31.80 \\
\hline $74^{\mathrm{a}}$ & Chi-Chi, Taiwan & 1999 & TCU068 & 191.15 & 3 & 1.34 & 0.082 & 38.35 \\
\hline 75 & Chi-Chi, Taiwan & 1999 & TCU075 & 72.17 & 4 & 3.73 & 0.213 & 29.42 \\
\hline 76 & Chi-Chi, Taiwan & 1999 & TCU076 & 39.31 & 4 & 3.51 & 0.262 & 28.29 \\
\hline 77 & Chi-Chi, Taiwan & 1999 & TCU082 & 39.28 & 4 & 1.45 & 0.133 & 35.33 \\
\hline $78^{\mathrm{a}}$ & Chi-Chi, Taiwan & 1999 & TCU087 & 31.81 & 4 & 4.14 & 0.111 & 41.67 \\
\hline 79 & Chi-Chi, Taiwan & 1999 & TCU098 & 26.68 & 4 & 5.53 & 0.158 & 48.69 \\
\hline 80 & Chi-Chi, Taiwan & 1999 & TCU101 & 39.18 & 4 & 3.63 & 0.146 & 17.07 \\
\hline 81 & Chi-Chi, Taiwan & 1999 & TCU102 & 57.71 & 4 & 1.05 & 0.159 & 36.76 \\
\hline 82 & Chi-Chi, Taiwan & 1999 & TCU103 & 54.46 & 4 & 4.97 & 0.135 & 39.55 \\
\hline $83^{\mathrm{a}}$ & Chi-Chi, Taiwan & 1999 & TCU104 & 25.36 & 4 & 0.00 & 0.083 & 42.11 \\
\hline 84 & Chi-Chi, Taiwan & 1999 & TCU128 & 72.22 & 4 & 2.48 & 0.177 & 45.29 \\
\hline 85 & Chi-Chi, Taiwan & 1999 & TCU136 & 36.77 & 4 & 4.17 & 0.113 & 42.55 \\
\hline 86 & Northwest China-03 & 1997 & Jiashi & 26.55 & 4 & 6.28 & 0.906 & 5.58 \\
\hline 87 & Yountville & 2000 & Napa Fire Station \#3 & 37.86 & 4 & 4.78 & 1.497 & 13.15 \\
\hline 88 & Chi-Chi, Taiwan-03 & 1999 & CHY024 & 32.07 & 4 & 0 & 0.335 & 13.59 \\
\hline 89 & Chi-Chi, Taiwan-03 & 1999 & CHY080 & 66.33 & 5.379 & 1.47 & 0.898 & 11 \\
\hline 90 & Chi-Chi, Taiwan-03 & 1999 & TCU076 & 45.39 & 4 & 4 & 1.179 & 10.43 \\
\hline 91 & Chi-Chi, Taiwan-06 & 1999 & CHY101 & 33.88 & 4.855 & 1.19 & 0.415 & 25.89 \\
\hline
\end{tabular}

a These records are extracted and simulated in different parameter ranges than those in Table 1. 

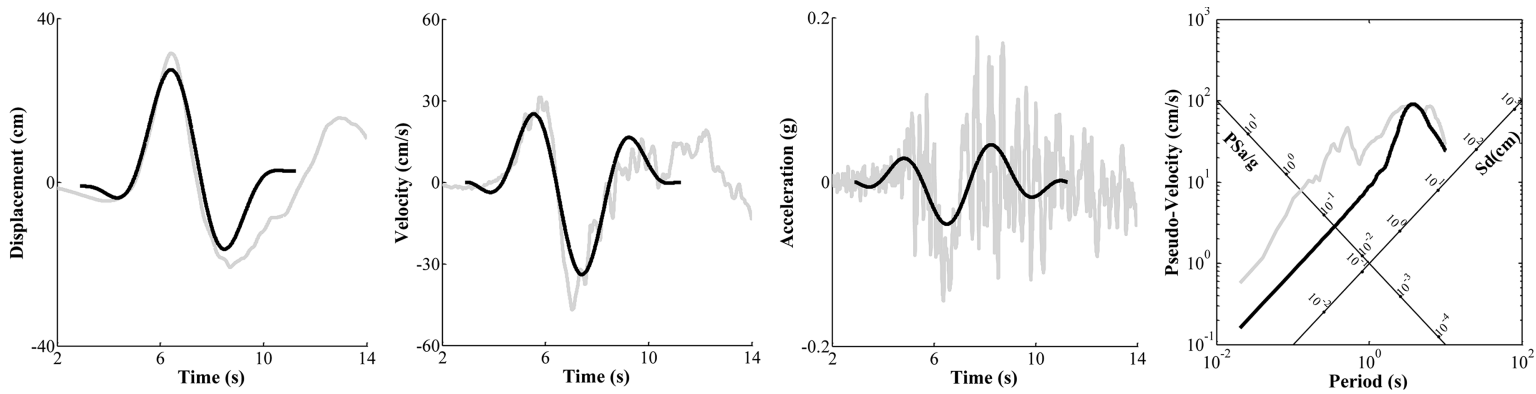

1979 Imperial Valley-06, Station El Centro Array \#10
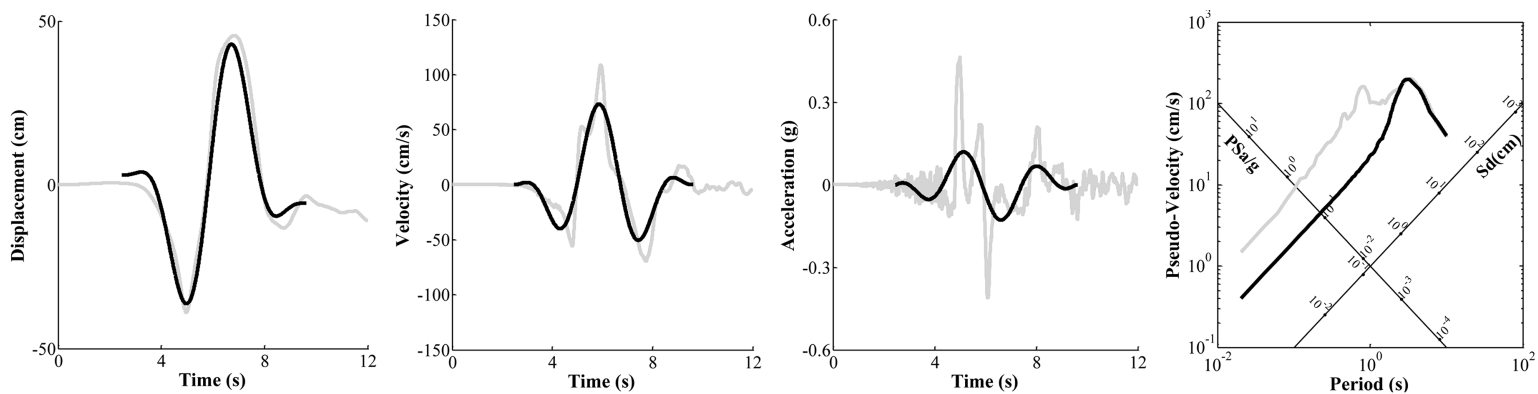

1979 Imperial Valley-06, Station El Centro Array \#7
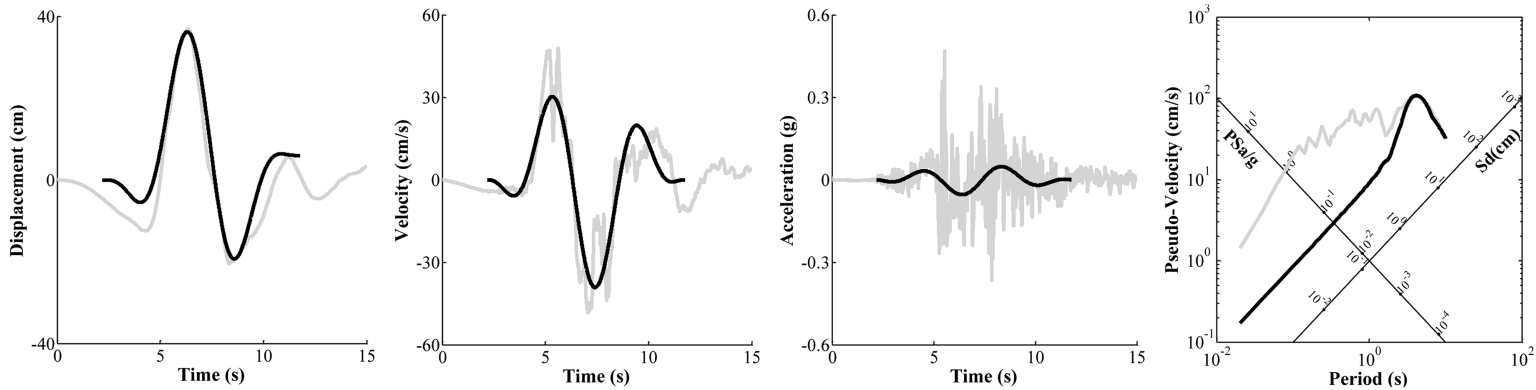

1979 Imperial Valley-06, Station El Centro Array \#8
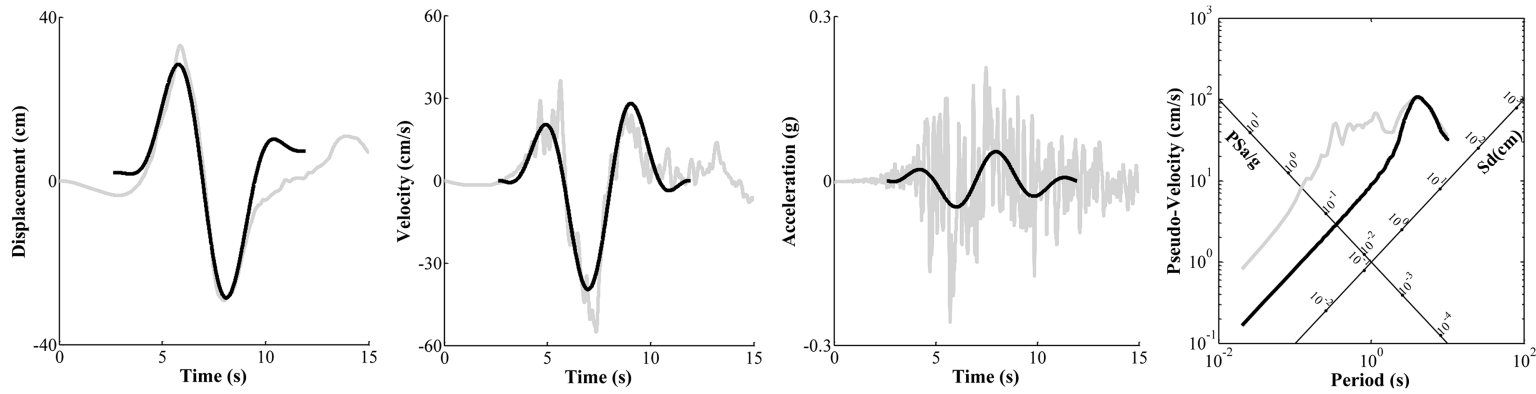

1979 Imperial Valley-06, Station Holtville Post Office
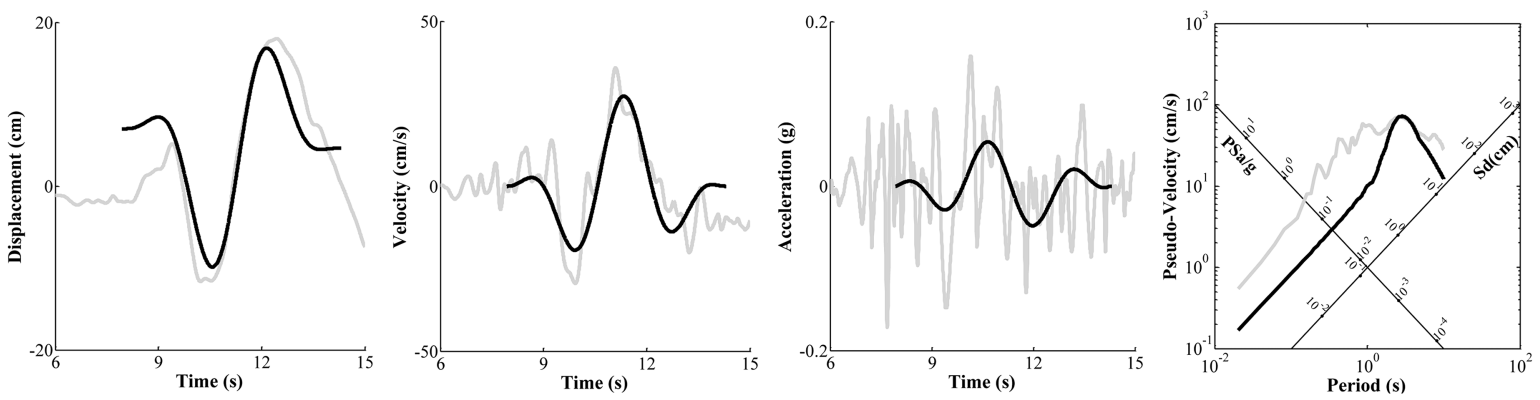

1981 Westmorland, Station Parachute Test Site

Figure 4. Displacement, velocity, acceleration, and tripartite logarithmic response spectra of actual record (gray) and those of simulated pulse (black) with a $5 \%$ damping ratio. 

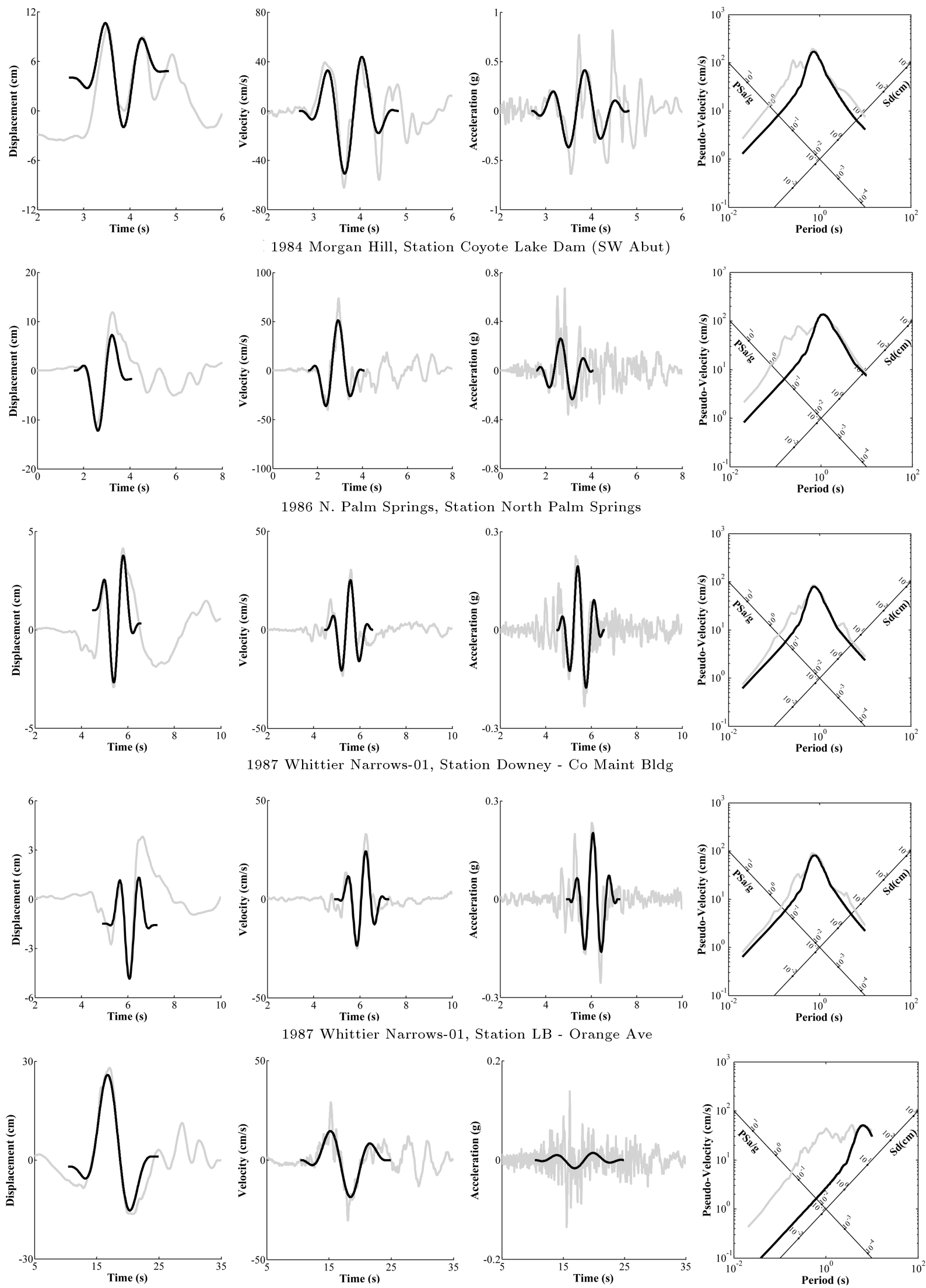

Figure 4. Displacement, velocity, acceleration, and tripartite logarithmic response spectra of actual record (gray) and those of simulated pulse (black) with a $5 \%$ damping ratio (continued). 

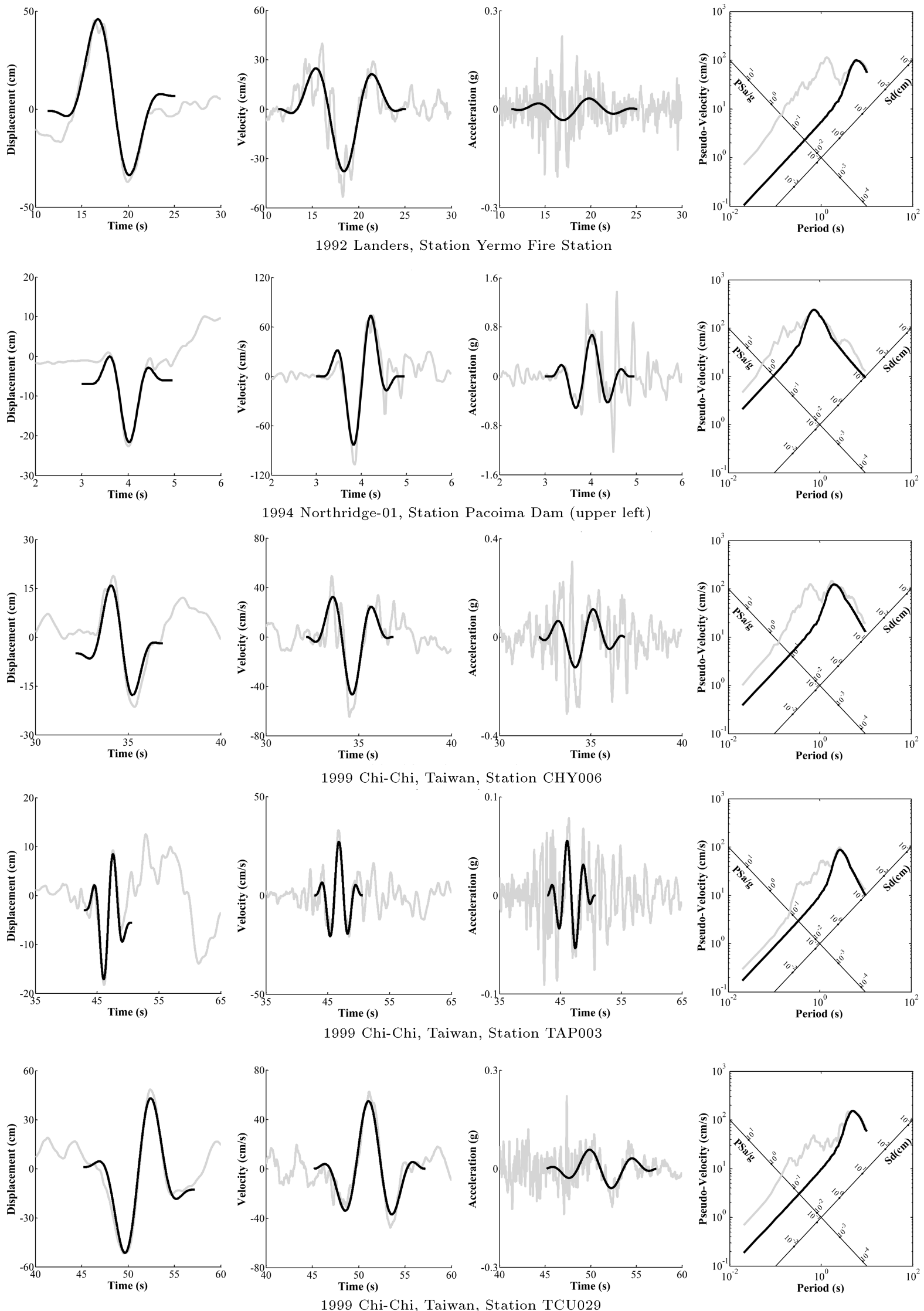

Figure 4. Displacement, velocity, acceleration, and tripartite logarithmic response spectra of actual record (gray) and those of simulated pulse (black) with a $5 \%$ damping ratio (continued). 

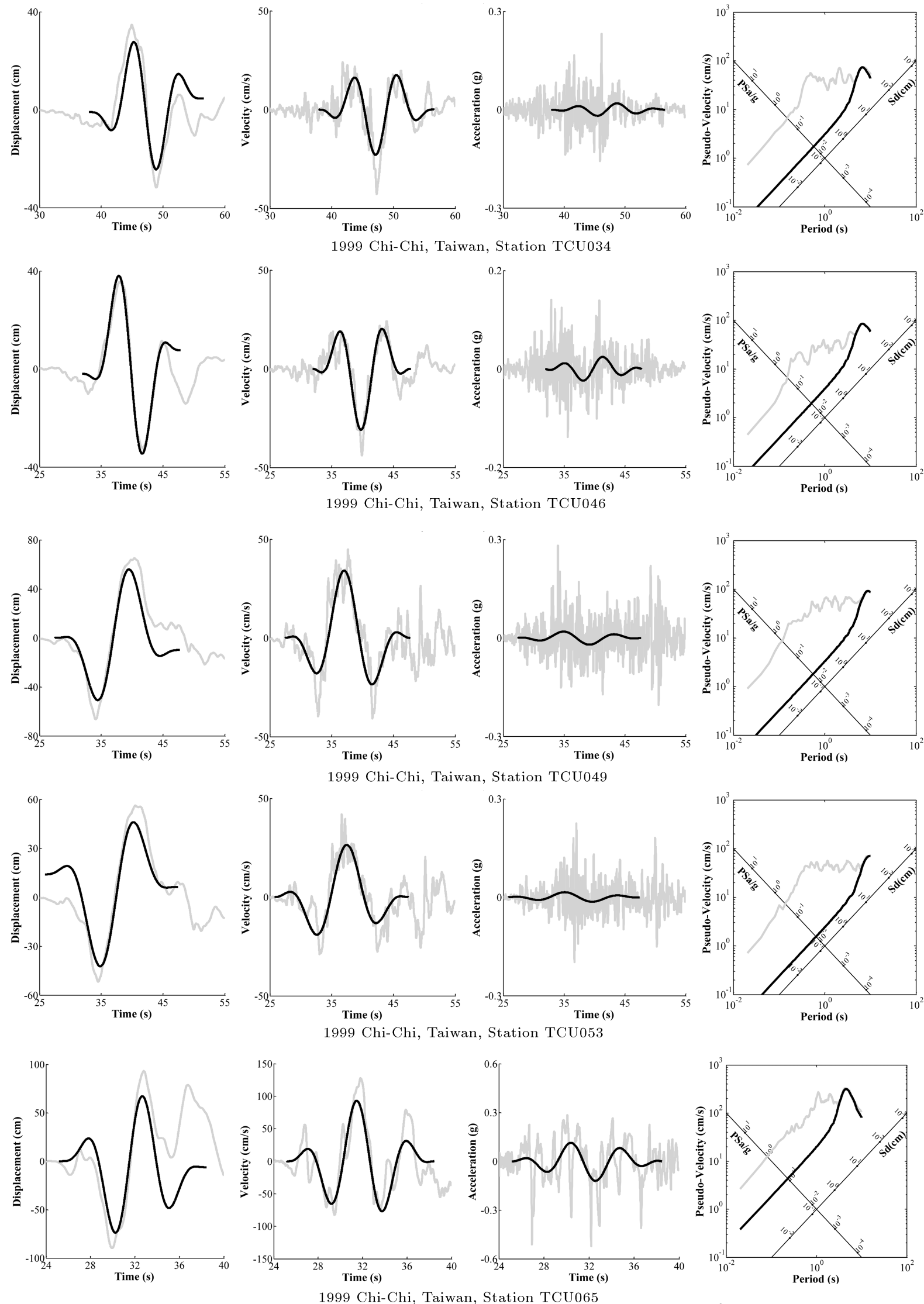

Figure 4. Displacement, velocity, acceleration, and tripartite logarithmic response spectra of actual record (gray) and those of simulated pulse (black) with a $5 \%$ damping ratio (continued). 

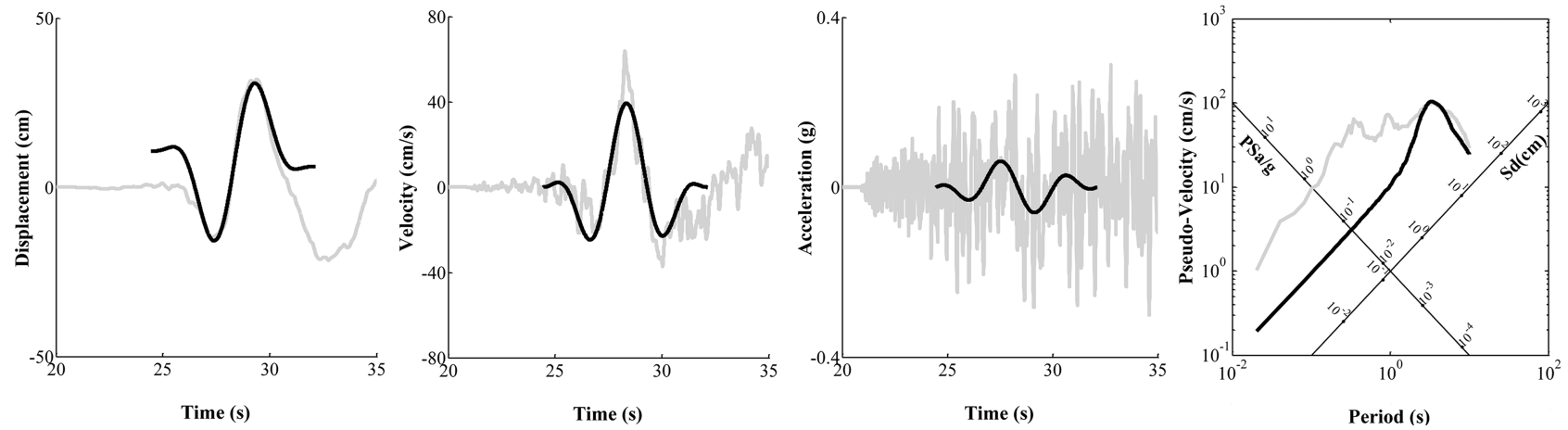

1999 Chi-Chi, Taiwan, Station TCU076
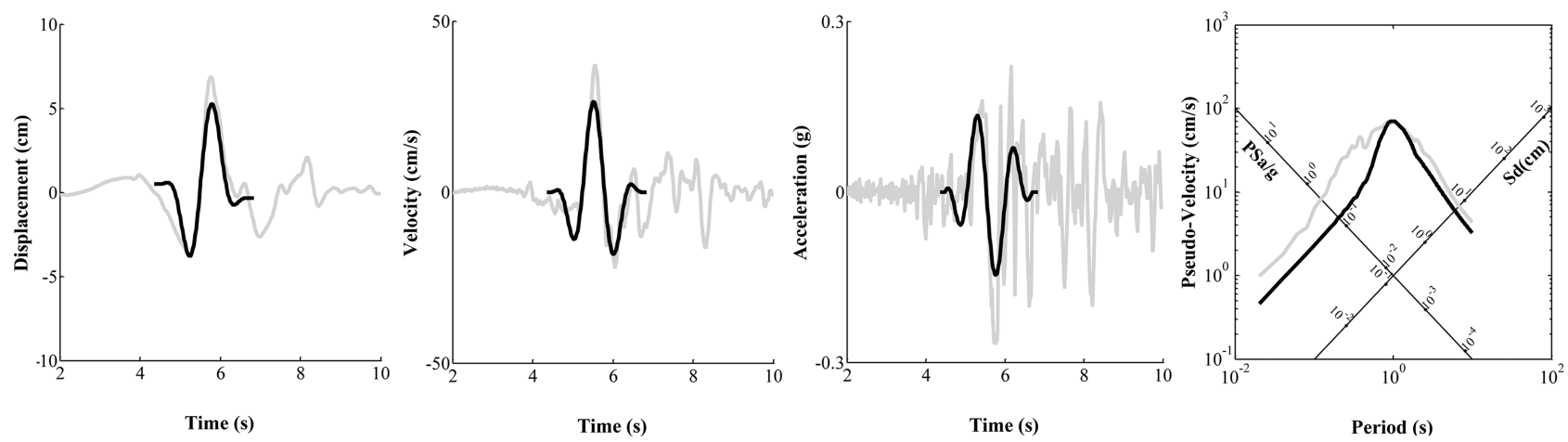

1997 Northwest China-03, Station Jiashi
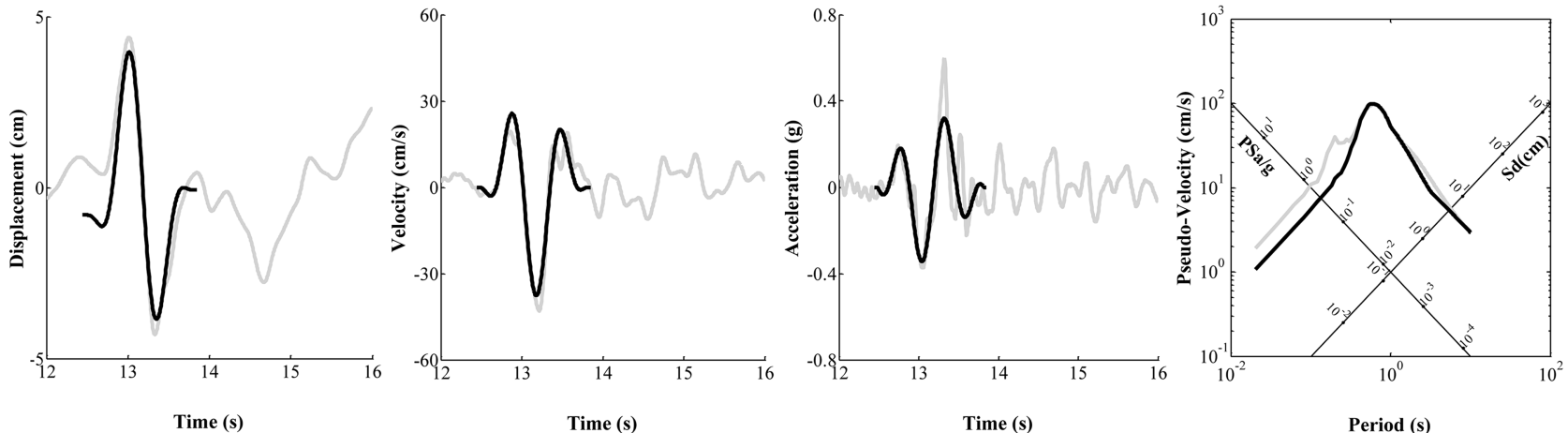

2000 Yountville, Station Napa Fire Station \#3
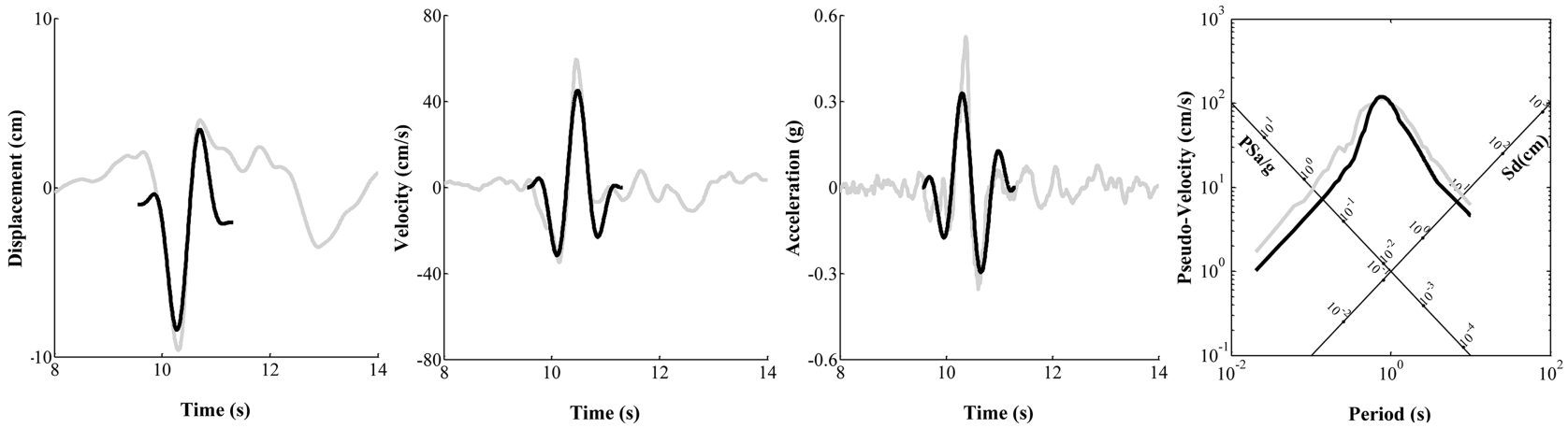

1999 Chi-Chi, Taiwan-03, Station TCU076

Figure 4. Displacement, velocity, acceleration, and tripartite logarithmic response spectra of actual record (gray) and those of simulated pulse (black) with a $5 \%$ damping ratio (continued). 
Table 5. The comparison between CSV of the actual record and the simulated pulse.

\begin{tabular}{|c|c|c|c|c|c|c|c|c|}
\hline No. & Event & Year & Station name & $\begin{array}{c}E_{\text {Record }} \\
\left(\mathrm{cm}^{2} / \mathrm{s}\right)\end{array}$ & $\begin{array}{c}E_{P R} \\
\left(\mathrm{~cm}^{2} / \mathrm{s}\right)\end{array}$ & $\begin{array}{c}E_{\mathrm{Pulse}} \\
\left(\mathrm{cm}^{2} / \mathrm{s}\right)\end{array}$ & $\frac{E_{\mathrm{PR}}}{E_{\text {Record }}}$ & $\frac{E_{\text {Pulse }}}{E_{\text {Record }}}$ \\
\hline 1 & San Fernando & 1971 & Pacoima Dam (upper left abut) & 9797 & 5578 & 4843 & 0.569 & 0.494 \\
\hline 2 & Coyote Lake & 1979 & Gilroy array \#6 & 760.6 & 673.9 & 535.6 & 0.886 & 0.704 \\
\hline 3 & Imperial Valley-06 & 1979 & Aeropuerto Mexicali & 1602 & 944.2 & 872.7 & 0.589 & 0.545 \\
\hline 4 & Imperial Valley-06 & 1979 & Agrarias & 1963 & 1485 & 1246 & 0.756 & 0.635 \\
\hline 5 & Imperial Valley-06 & 1979 & Brawley Airport & 2105 & 1384 & 1202 & 0.657 & 0.571 \\
\hline 6 & Imperial Valley-06 & 1979 & EC County Center FF & 4559 & 3525 & 2792 & 0.773 & 0.612 \\
\hline 7 & Imperial Valley-06 & 1979 & EC Meloland Overpass FF & 9448 & 8464 & 7076 & 0.896 & 0.749 \\
\hline 8 & Imperial Valley-06 & 1979 & El Centro array \#10 & 3883 & 2599 & 2015 & 0.669 & 0.519 \\
\hline 9 & Imperial Valley-06 & 1979 & El Centro array \#11 & 2042 & 1690 & 1001 & 0.828 & 0.49 \\
\hline 10 & Imperial Valley-06 & 1979 & El Centro array \#3 & 2129 & 1711 & 1353 & 0.804 & 0.636 \\
\hline 11 & Imperial Valley-06 & 1979 & El Centro array \#4 & 9550 & 8877 & 7592 & 0.930 & 0.795 \\
\hline 12 & Imperial Valley-06 & 1979 & El Centro array \#5 & 12194 & 10740 & 9285 & 0.881 & 0.761 \\
\hline 13 & Imperial Valley-06 & 1979 & El Centro array \#6 & 15171 & 14472 & 13476 & 0.954 & 0.888 \\
\hline 14 & Imperial Valley-06 & 1979 & El Centro array \#7 & 10326 & 9813 & 7804 & 0.950 & 0.756 \\
\hline 15 & Imperial Valley-06 & 1979 & El Centro array \#8 & 4316 & 3886 & 3083 & 0.900 & 0.714 \\
\hline 16 & Imperial Valley-06 & 1979 & El Centro differential array & 4755 & 4050 & 2489 & 0.852 & 0.524 \\
\hline 17 & Imperial Valley-06 & 1979 & Holtville Post Office & 3890 & 3480 & 3021 & 0.894 & 0.777 \\
\hline 18 & Mammoth Lakes-06 & 1980 & Long Valley Dam (Upr L abut) & 544.9 & 376.5 & 271.5 & 0.691 & 0.498 \\
\hline 19 & Irpinia, Italy-01 & 1980 & Sturno & 3184 & 2188 & 2000 & 0.687 & 0.628 \\
\hline 20 & Westmorland & 1981 & Parachute Test Site & 2041 & 1343 & 975.8 & 0.658 & 0.478 \\
\hline 21 & Coalinga-0 05 & 1983 & Oil City & 827.4 & 456.5 & 405.4 & 0.552 & 0.49 \\
\hline 22 & Coalinga-0 05 & 1983 & Transmitter Hill & 1334 & 1128 & 872.3 & 0.846 & 0.654 \\
\hline 23 & Coalinga-07 & 1983 & Coalinga-14th \& Elm (Old CHP) & 328.2 & 267.3 & 191.7 & 0.814 & 0.584 \\
\hline 24 & Morgan Hill & 1984 & Coyote Lake Dam (SW Abut) & 1876 & 1507 & 1131 & 0.803 & 0.603 \\
\hline 25 & Morgan Hill & 1984 & Gilroy array \#6 & 730.8 & 557.2 & 405.2 & 0.763 & 0.554 \\
\hline 26 & Taiwan SMART1(40) & 1986 & SMART1 C00 & 882.2 & 524.2 & 538.4 & 0.594 & 0.610 \\
\hline 27 & Taiwan SMART1(40) & 1986 & SMART1 M07 & 880.9 & 713 & 726 & 0.809 & 0.824 \\
\hline 28 & N. Palm Springs & 1986 & North Palm Springs & 1964 & 1572 & 1334 & 0.800 & 0.679 \\
\hline 29 & San Salvador & 1986 & Geotech Investig Center & 2379 & 1427 & 1220 & 0.600 & 0.513 \\
\hline 30 & Whittier Narrows-01 & 1987 & Downey - Co Maint Bldg & 370.9 & 315.5 & 268.5 & 0.850 & 0.724 \\
\hline 31 & Whittier Narrows-01 & 1987 & LB - Orange Ave & 478 & 378.1 & 283.7 & 0.791 & 0.593 \\
\hline
\end{tabular}


Table 5. The comparison between CSV of the actual record and the simulated pulse (continued).

\begin{tabular}{|c|c|c|c|c|c|c|c|c|}
\hline No. & Event & Year & Station name & $\begin{array}{c}E_{\text {Record }} \\
\left(\mathrm{cm}^{2} / \mathrm{s}\right)\end{array}$ & $\begin{array}{c}E_{P R} \\
\left(\mathrm{~cm}^{2} / \mathrm{s}\right)\end{array}$ & $\begin{array}{c}E_{\text {Pulse }} \\
\left(\mathrm{cm}^{2} / \mathrm{s}\right)\end{array}$ & $\frac{E_{\mathrm{PR}}}{E_{\text {Record }}}$ & $\frac{E_{\text {Pulse }}}{E_{\text {Record }}}$ \\
\hline 32 & Superstition Hills-02 & 1987 & Parachute Test Site & 12863 & 9462 & 7332 & 0.736 & 0.57 \\
\hline 33 & Loma Prieta & 1989 & Alameda Naval Air Stn Hanger & 1029 & 702.5 & 434.4 & 0.683 & 0.422 \\
\hline 34 & Loma Prieta & 1989 & Gilroy array \#2 & 1956 & 1489 & 1126 & 0.761 & 0.576 \\
\hline 35 & Loma Prieta & 1989 & Oakland - Outer Harbor Wharf & 1825 & 1358 & 959.8 & 0.744 & 0.526 \\
\hline 36 & Loma Prieta & 1989 & Saratoga - Aloha Ave & 3334 & 2504 & 1196 & 0.751 & 0.359 \\
\hline 37 & Erzican, Turkey & 1992 & Erzincan & 7981 & 6867 & 4969 & 0.860 & 0.623 \\
\hline 38 & Cape Mendocino & 1992 & Petrolia & 4631 & 3808 & 2046 & 0.822 & 0.442 \\
\hline 39 & Landers & 1992 & Barstow & 1835 & 1456 & 1062 & 0.793 & 0.579 \\
\hline 40 & Landers & 1992 & Lucerne & 21852 & 21319 & 11956 & 0.976 & 0.547 \\
\hline 41 & Landers & 1992 & Yermo Fire Station & 5650 & 5308 & 3958 & 0.940 & 0.701 \\
\hline 42 & Northridge-01 & 1994 & Jensen Filter Plant & 7842 & 6530 & 5635 & 0.833 & 0.719 \\
\hline 43 & Northridge-01 & 1994 & Jensen Filter Plant Generator & 7869 & 6534 & 5625 & 0.830 & 0.715 \\
\hline 44 & Northridge-01 & 1994 & LA-Wadsworth VA Hospital North & 1118 & 566.7 & 303.8 & 0.507 & 0.272 \\
\hline 45 & Northridge-01 & 1994 & LA Dam & 3604 & 2392 & 1541 & 0.664 & 0.428 \\
\hline 46 & Northridge-01 & 1994 & Newhall - W Pico Canyon Rd. & 8977 & 7984 & 5841 & 0.889 & 0.651 \\
\hline 47 & Northridge-01 & 1994 & Pacoima Dam (downstr) & 820.7 & 411.6 & 235.4 & 0.501 & 0.287 \\
\hline 48 & Northridge-01 & 1994 & Pacoima Dam (upper left) & 5265 & 3233 & 2682 & 0.614 & 0.509 \\
\hline 49 & Northridge-01 & 1994 & Rinaldi Receiving Sta & 12279 & 8850 & 7313 & 0.721 & 0.596 \\
\hline 50 & Northridge-01 & 1994 & Sylmar - Converter Sta & 19938 & 13005 & 8695 & 0.652 & 0.436 \\
\hline 51 & Northridge-01 & 1994 & Sylmar - Converter Sta east & 8348 & 6071 & 4664 & 0.727 & 0.559 \\
\hline 52 & Northridge-01 & 1994 & Sylmar - Olive view med FF & 10067 & 7203 & 5560 & 0.715 & 0.552 \\
\hline 53 & Kobe, Japan & 1995 & Takarazuka & 3716 & 2893 & 2107 & 0.778 & 0.567 \\
\hline 54 & Kobe, Japan & 1995 & Takatori & 31747 & 18343 & 11261 & 0.578 & 0.355 \\
\hline 55 & Kocaeli, Turkey & 1999 & Gebze & 4835 & 4383 & 3151 & 0.907 & 0.652 \\
\hline 56 & Chi-Chi, Taiwan & 1999 & CHY006 & 5585 & 3066 & 2090 & 0.549 & 0.374 \\
\hline 57 & Chi-Chi, Taiwan & 1999 & CHY035 & 2371 & 1297 & 865.1 & 0.547 & 0.365 \\
\hline 58 & Chi-Chi, Taiwan & 1999 & CHY101 & 12935 & 9017 & 6685 & 0.697 & 0.517 \\
\hline 59 & Chi-Chi, Taiwan & 1999 & TAP003 & 2371 & 1395 & 1162 & 0.588 & 0.490 \\
\hline 60 & Chi-Chi, Taiwan & 1999 & TCU029 & 9457 & 8277 & 7293 & 0.875 & 0.771 \\
\hline
\end{tabular}


Table 5. The comparison between CSV of the actual record and the simulated pulse (continued).

\begin{tabular}{|c|c|c|c|c|c|c|c|c|}
\hline No. & Event & Year & Station name & $\begin{array}{c}E_{\text {Record }} \\
\left(\mathrm{cm}^{2} / \mathrm{s}\right)\end{array}$ & $\begin{array}{c}E_{P R} \\
\left(\mathrm{~cm}^{2} / \mathrm{s}\right)\end{array}$ & $\begin{array}{c}E_{\text {Pulse }} \\
\left(\mathrm{cm}^{2} / \mathrm{s}\right)\end{array}$ & $\frac{E_{\mathrm{PR}}}{E_{\text {Record }}}$ & $\frac{E_{\text {Pulse }}}{E_{\text {Record }}}$ \\
\hline 61 & Chi-Chi, Taiwan & 1999 & TCU031 & 11468 & 9321 & 8506 & 0.813 & 0.742 \\
\hline 62 & Chi-Chi, Taiwan & 1999 & TCU034 & 3445 & 3207 & 1978 & 0.931 & 0.574 \\
\hline 63 & Chi-Chi, Taiwan & 1999 & TCU036 & 11581 & 9185 & 7313 & 0.793 & 0.631 \\
\hline 64 & Chi-Chi, Taiwan & 1999 & TCU038 & 9329 & 6716 & 6164 & 0.720 & 0.661 \\
\hline 65 & Chi-Chi, Taiwan & 1999 & TCU040 & 7806 & 6720 & 5316 & 0.861 & 0.681 \\
\hline 66 & Chi-Chi, Taiwan & 1999 & TCU042 & 4941 & 3755 & 2990 & 0.760 & 0.605 \\
\hline 67 & Chi-Chi, Taiwan & 1999 & TCU046 & 3722 & 3438 & 3077 & 0.924 & 0.827 \\
\hline 68 & Chi-Chi, Taiwan & 1999 & TCU049 & 7657 & 6697 & 4833 & 0.875 & 0.631 \\
\hline 69 & Chi-Chi, Taiwan & 1999 & TCU053 & 5217 & 4229 & 3136 & 0.811 & 0.601 \\
\hline 70 & Chi-Chi, Taiwan & 1999 & TCU054 & 8964 & 7334 & 4504 & 0.818 & 0.502 \\
\hline 71 & Chi-Chi, Taiwan & 1999 & TCU056 & 5660 & 4663 & 2109 & 0.824 & 0.373 \\
\hline 72 & Chi-Chi, Taiwan & 1999 & TCU060 & 4034 & 3633 & 2366 & 0.901 & 0.587 \\
\hline 73 & Chi-Chi, Taiwan & 1999 & TCU065 & 48055 & 33512 & 23496 & 0.697 & 0.489 \\
\hline 74 & Chi-Chi, Taiwan & 1999 & TCU068 & 161270 & 151168 & 135657 & 0.937 & 0.841 \\
\hline 75 & Chi-Chi, Taiwan & 1999 & TCU075 & 17952 & 13447 & 9921 & 0.749 & 0.553 \\
\hline 76 & Chi-Chi, Taiwan & 1999 & TCU076 & 5995 & 3224 & 2398 & 0.538 & 0.400 \\
\hline 77 & Chi-Chi, Taiwan & 1999 & TCU082 & 9468 & 7376 & 4720 & 0.779 & 0.499 \\
\hline 78 & Chi-Chi, Taiwan & 1999 & TCU087 & 6287 & 5947 & 3710 & 0.946 & 0.590 \\
\hline 79 & Chi-Chi, Taiwan & 1999 & TCU098 & 4233 & 2564 & 1835 & 0.606 & 0.433 \\
\hline 80 & Chi-Chi, Taiwan & 1999 & TCU101 & 8733 & 7001 & 4263 & 0.802 & 0.488 \\
\hline 81 & Chi-Chi, Taiwan & 1999 & TCU102 & 19241 & 14996 & 8546 & 0.779 & 0.444 \\
\hline 82 & Chi-Chi, Taiwan & 1999 & TCU103 & 12068 & 10360 & 8953 & 0.859 & 0.742 \\
\hline 83 & Chi-Chi, Taiwan & 1999 & TCU104 & 4921 & 4055 & 3150 & 0.824 & 0.640 \\
\hline 84 & Chi-Chi, Taiwan & 1999 & TCU128 & 17524 & 14231 & 11969 & 0.812 & 0.683 \\
\hline 85 & Chi-Chi, Taiwan & 1999 & TCU136 & 7147 & 6277 & 4872 & 0.878 & 0.682 \\
\hline 86 & Northwest China-03 & 1997 & Jiashi & 542.6 & 421.5 & 316.5 & 0.777 & 0.583 \\
\hline 87 & Yountville & 2000 & Napa Fire Station \#3 & 531 & 406.8 & 389.7 & 0.766 & 0.734 \\
\hline 88 & Chi-Chi, Taiwan-03 & 1999 & CHY024 & 2244 & 1719 & 1250 & 0.766 & 0.557 \\
\hline 89 & Chi-Chi, Taiwan-03 & 1999 & CHY080 & 3222 & 2982 & 2678 & 0.926 & 0.831 \\
\hline 90 & Chi-Chi, Taiwan-03 & 1999 & TCU076 & 1012 & 886.6 & 711.3 & 0.876 & 0.703 \\
\hline 91 & Chi-Chi, Taiwan-06 & 1999 & CHY101 & 2179 & 1474 & 1363 & 0.677 & 0.626 \\
\hline
\end{tabular}




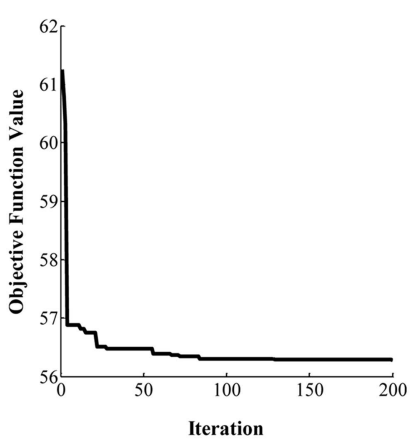

1979 Imperial Valley-06, Station El Centro Array \#10

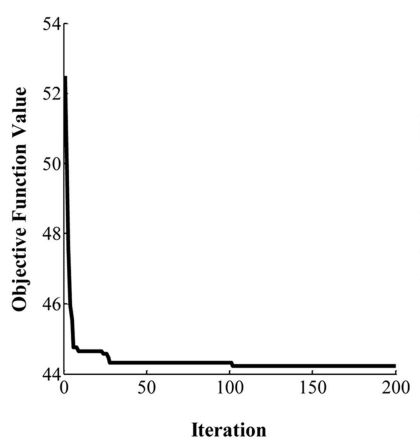

1981 Westmorland, Station Parachute Test Site

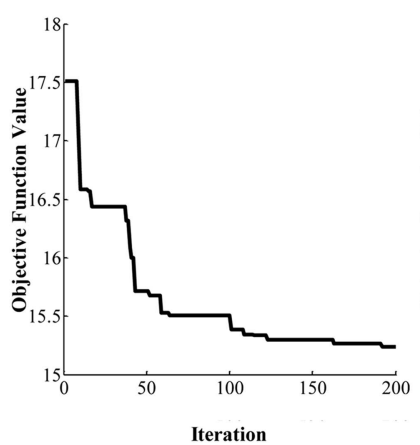

1987 Whittier Narrows-01, Station LB-Orange Ave

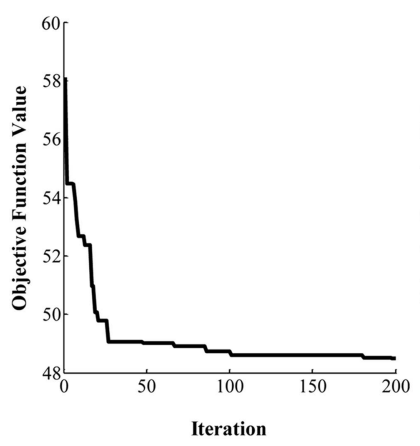

1999 Chi-Chi, Taiwan, Station CHY006
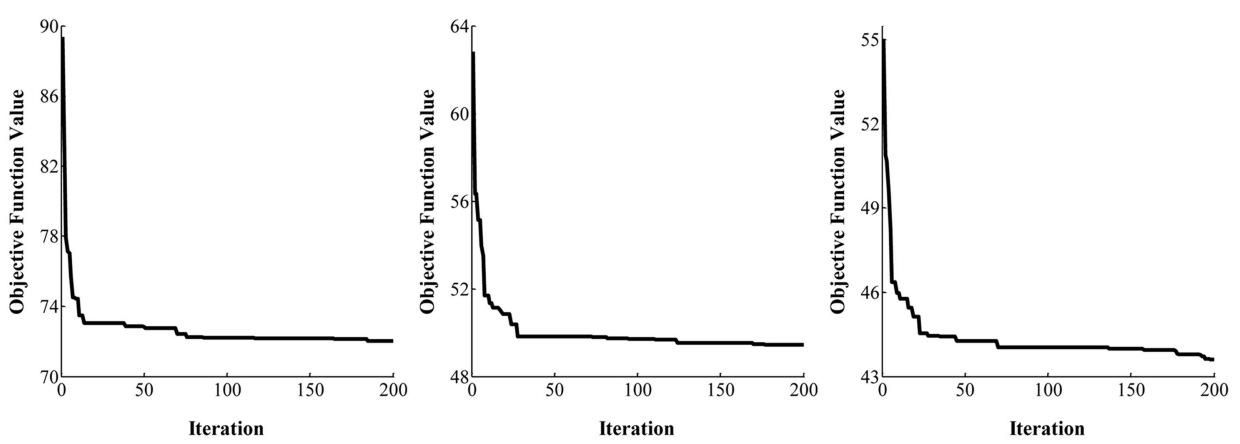

1979 Imperial Valley-06 Station El Centro Array \#7

1979 Imperial Valley-06, Station El Centro Array \#8

1979 Imperial Valley-06, Station Holtville Post Office
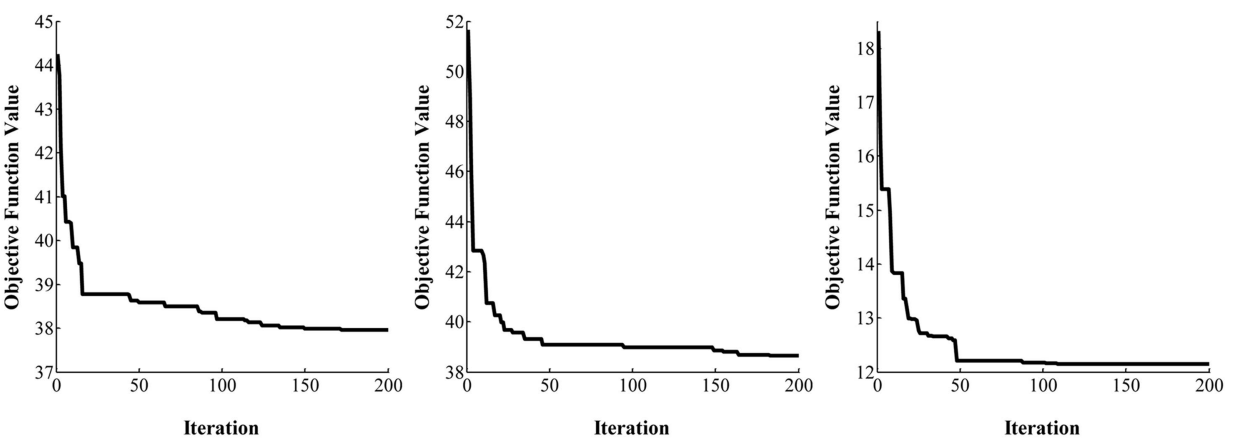

1984 Morgan Hill, Station

1986 N. Palm Springs, Station North Palm Springs

1987 Whittier Narrows-01, Station Downey - Co Maint Bldg:
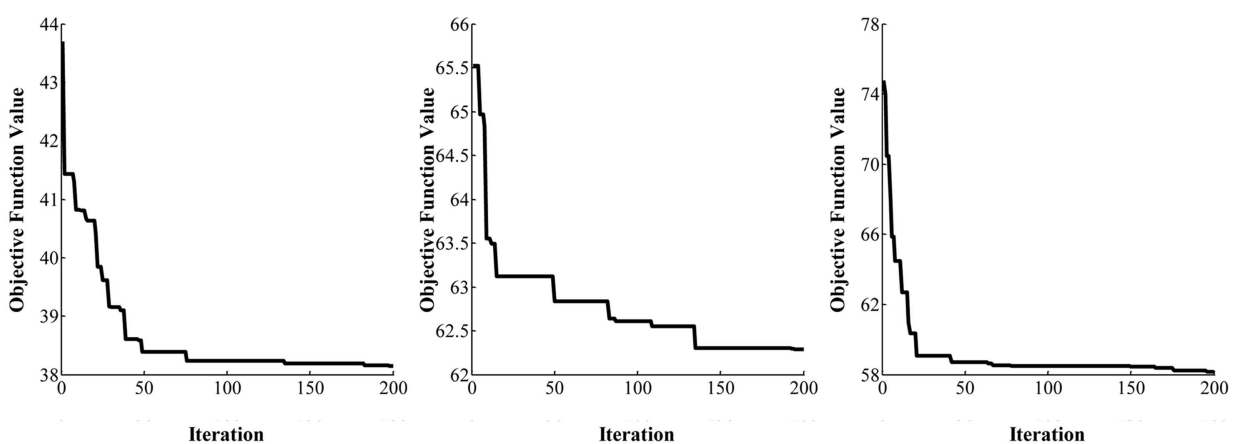

1992 Landers, Station Barstow

1992 Landers, Station Yermo Fire Station

1994 Northridge-01 - Station Pacoima Dam (upper left)
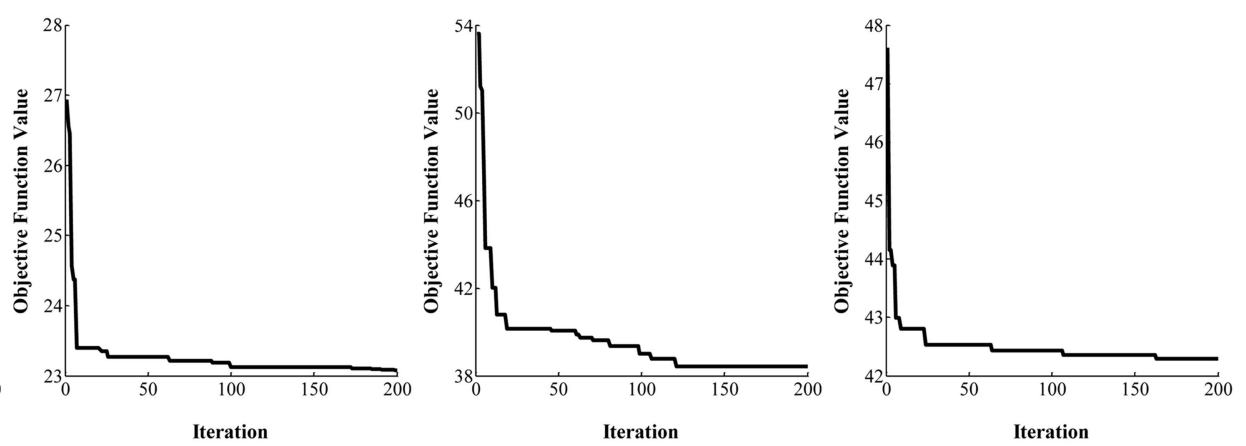

1999 Chi-Chi, Taiwan, Station TAP003

Chi, Taiwan, Station TCU029

1999 Chi-Chi, Taiwan, Station TCU034

Figure 5. The convergence of the proposed approach. 


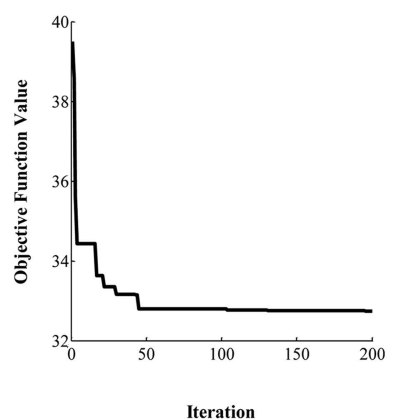

1999 Chi-Chi, Taiwan, Station TCU046

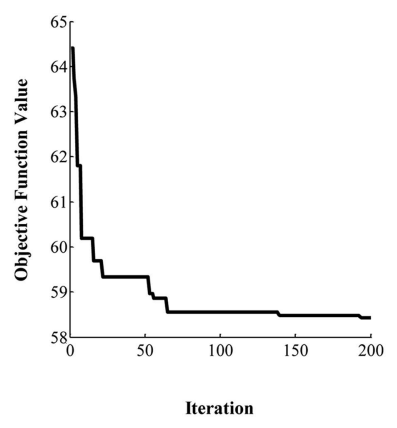

1999 Chi-Chi, Taiwan, Station TCU049

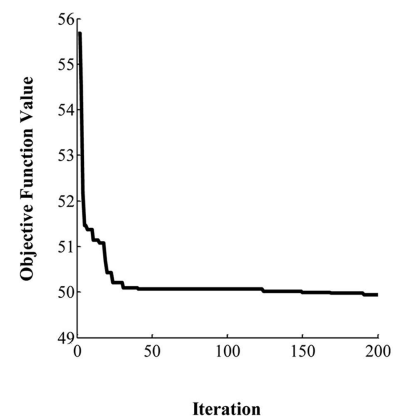

Iteration

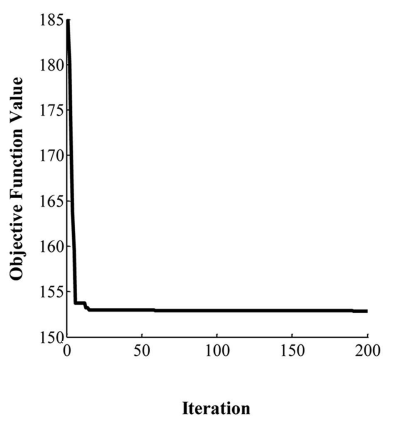

1999 Chi-Chi, Taiwan, Station TCU065

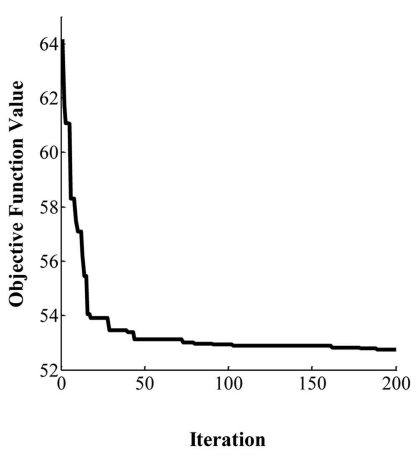

1999 Chi-Chi, Taiwan, Station TCU076

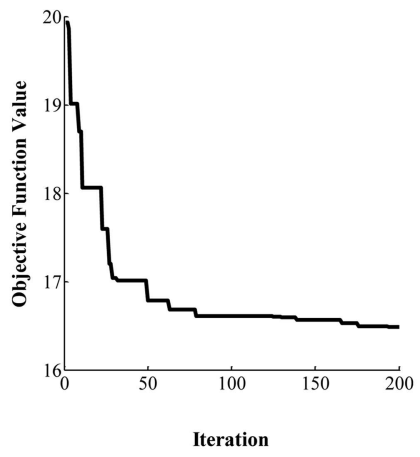

1997 Northwest China-03,

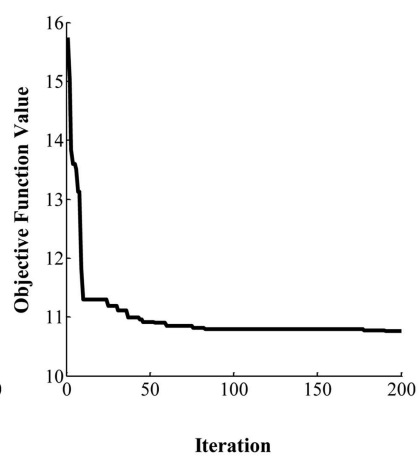

2000 Yountville, Station Napa, Fire Station \#3

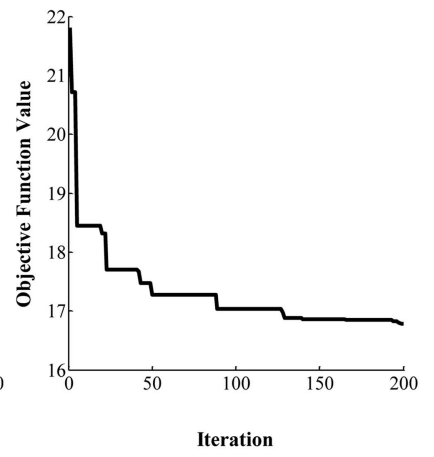

1999 Chi-Chi, Taiwan-03, Station TCU076

Figure 5. The convergence of the proposed approach (continued).

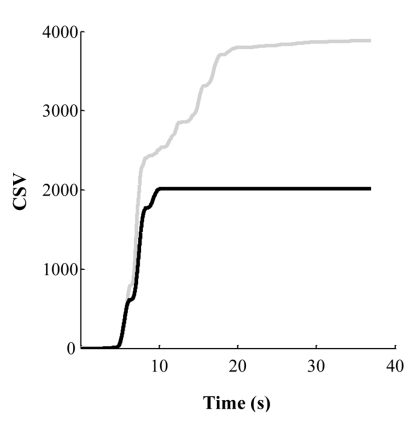

1979 Imperial Valley-06, Station El Centro Array \#10

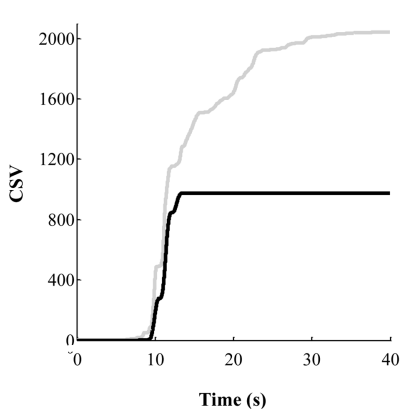

1981 Westmorland, Station Parachute Test Site

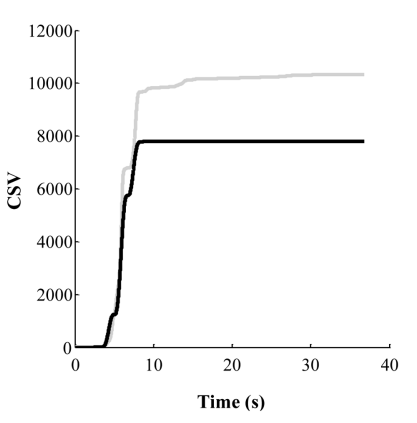

1979 Imperial Valley-06, Station El Centro Array \#7

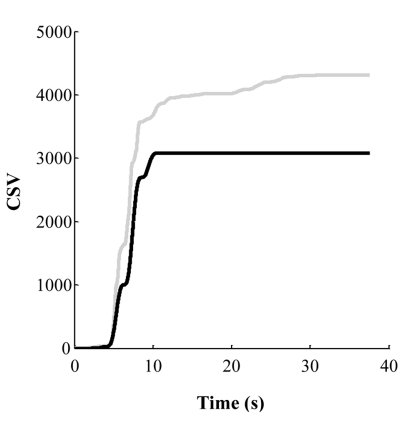

1979 Imperial Valley-06, Station El Centro Array \#8

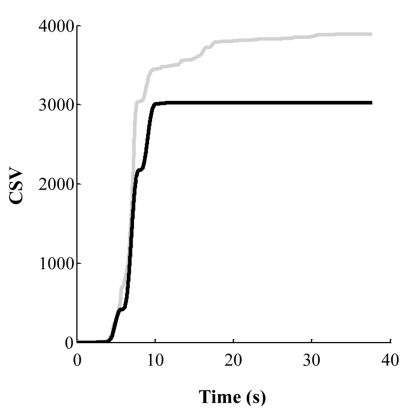

1979 Imperial Valley-06, Station Holtville Post Office

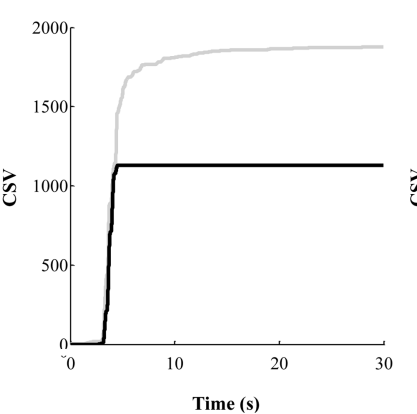

1984 Morgan Hill, Station Coyote Lake Dam (SW Abut)
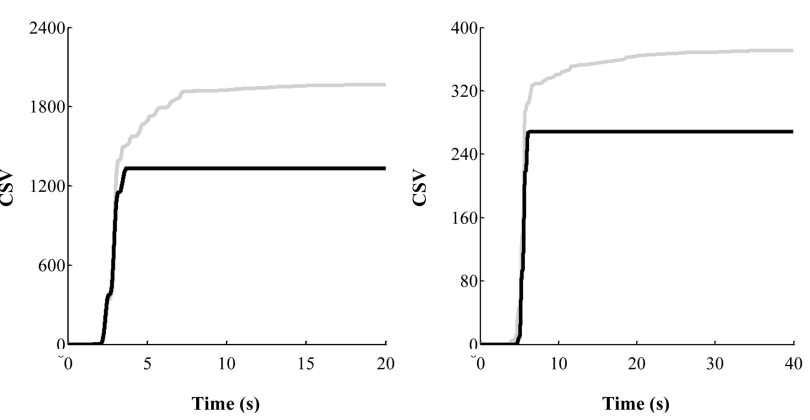

1986 N. Palm Springs, Station 1987 Whittier Narrows-01, Station North Palm Springs Downey - Co Maint Bldg

Figure 6. CSV of actual record (gray) and that of the simulated pulse (black). 


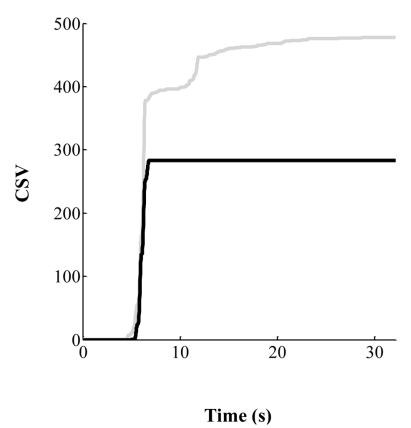

1987 Whittier Narrows-01, Station LB - Orange Ave

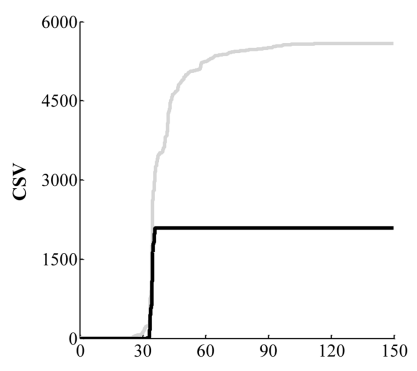

Time (s)

1999 Chi-Chi, Taiwan, Station CHY006

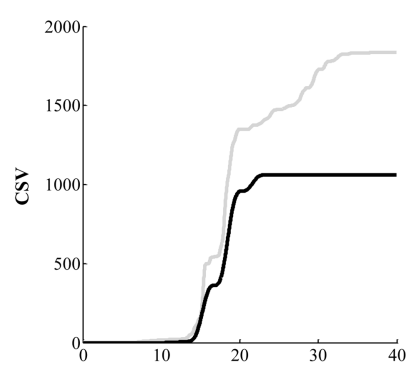

Time (s)

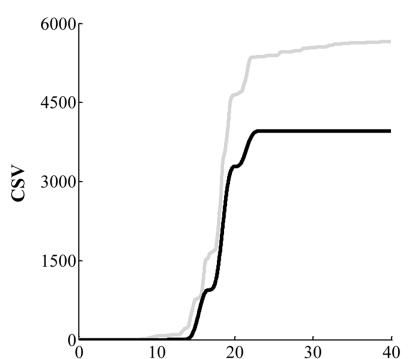

Time (s)

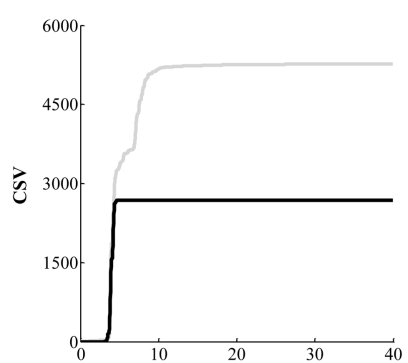

Time (s)

1992 Landers, Station Yermo Fire Station

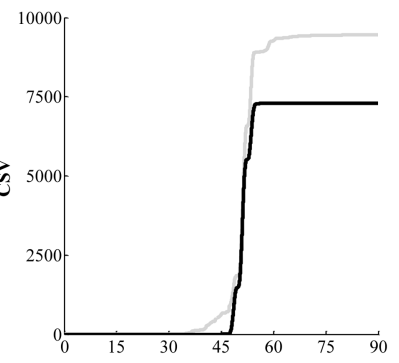

Time (s)

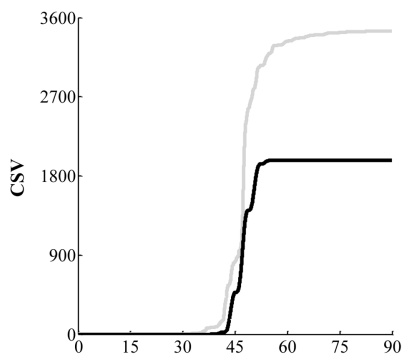

Time (s)

1999 Chi-Chi, Taiwan, Station 1999 Chi-Chi, Taiwan, Station TCU029

TCU034

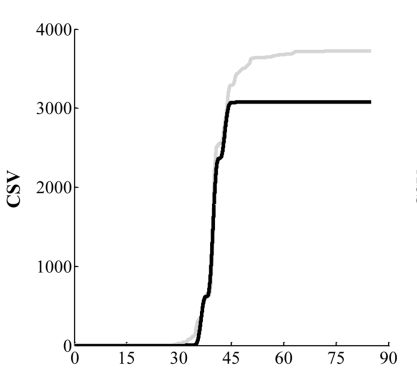

Time (s)

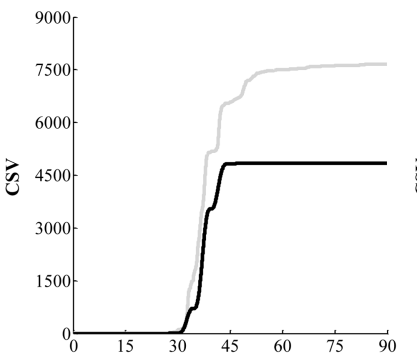

Time (s)

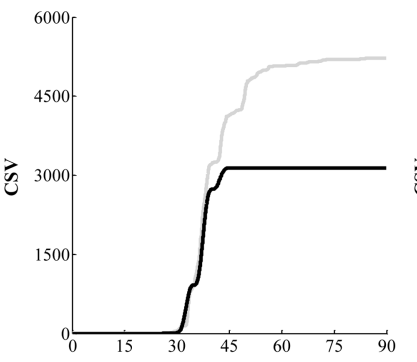

Time (s)

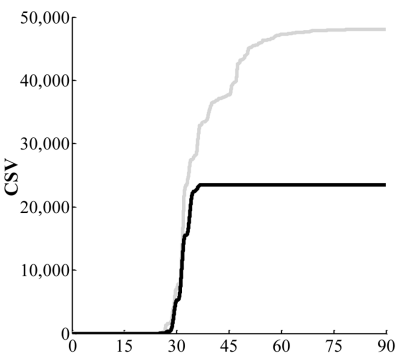

Time (s)

1999 Chi-Chi, Taiwan, Station I 1999 Chi-Chi, Taiwan, Station TCU046 TCU049

1999 Chi-Chi, Taiwan, Station TCU053

1999 Chi-Chi, Taiwan, Station TCU065

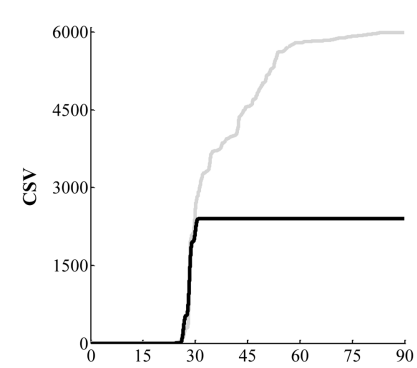

Time (s)

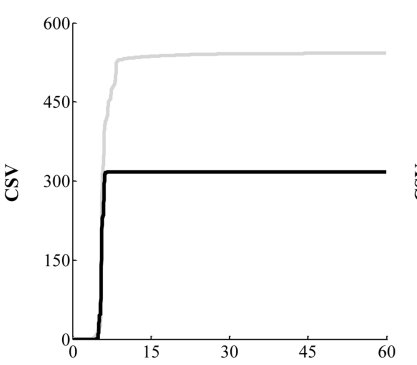

Time (s)

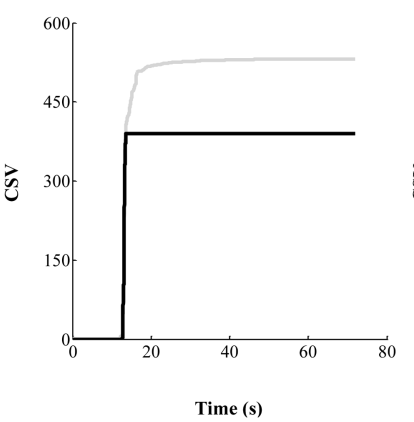

2000 Yountville, Station Napa Fire Station \#3

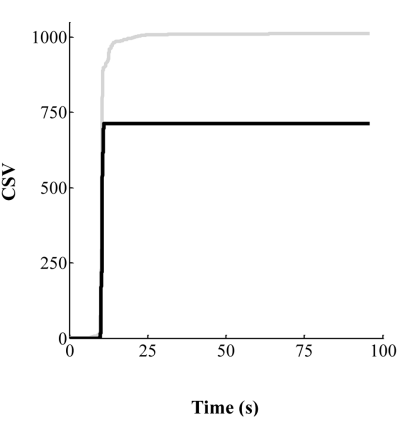

1999 Chi-Chi, Taiwan-03, Station TCU076

Figure 6. CSV of actual record (gray) and that of the simulated pulse (black) (continued). 


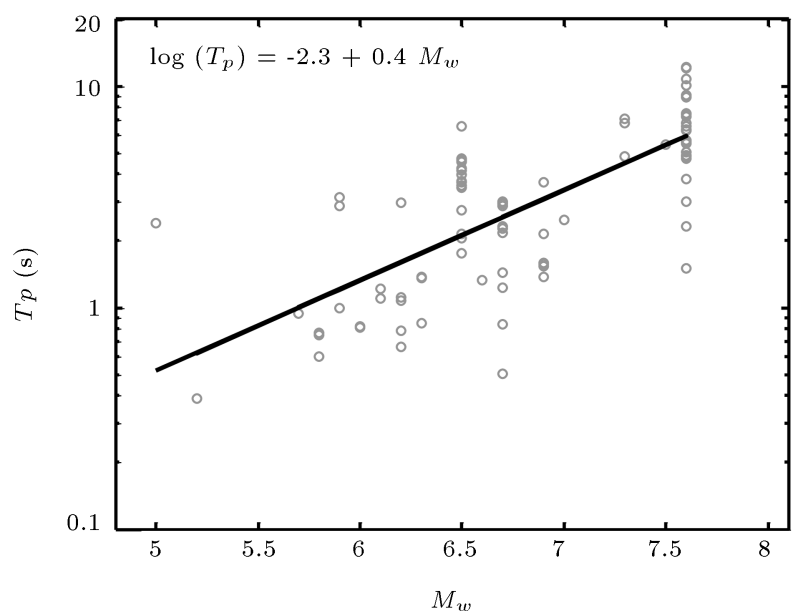

Figure 7. Linear regression of the prevalent pulse period and the earthquake magnitude.

\section{Conclusion}

Because of the destructive effects of near-fault earthquakesand pulse-like motions on structures and infrastructure, they have attracted special attention in seismology and earthquake engineering, and many analytical models have been presented to simulate such records. Some of these models are simple mathematical equations that use clear physical parameters to mathematically represent pulse-like records by simultaneously fitting time histories and response spectra of the actual records and the simulated pulses through a trialand-error process. Such a process limits the ability of researchers and engineers to practically use the simulation model. Therefore, the trial-and-error process was replaced in this study by using constrained PSO algorithm. In addition, by applying penalty function, rootmean-square differences of time histories and response spectra of the actual records and simulated pulses were minimized simultaneously. Then, a set of 91 pulse-like records from NGA strong ground motion database was studied, and by choosing a mathematical model and using the proposed approach, the largest velocity pulse in this set was identified, extracted, and represented mathematically. The results are presented in a table of parameters. This information can help en gineers study structure responses to pulse-like records. Moreover, the proposed approach can facilitate the extraction of any pulse-like record and its representation as closed mathematical equations. The recorded pulse history can be used for structural analysis and for studying structure responses to pulse-like motions.

\section{References}

1. Bertero, V.V., Mahin, S.A., and Herrera, R.A. "Aseismic design implications of near-fault San Fer- nando earthquake records", Earthquake Engineering and Structural Dynamics, 6(1), pp. 31-42 (1978).

2. Anderson, J.C. and Bertero, V.V. "Uncertainties in establishing design earthquakes", Journal of Structural Engineering, 113(8), pp. 1709-1724 (1987).

3. Hall, J.F., Heaton, T.H., Halling, M.W., and Wald, D.J. "Near-source ground motion and its effects on flexible buildings", Earthquake Spectra, 11(4), pp. 569605 (1995).

4. Iwan, W.D. "Drift spectrum: Measure of demand for earthquake ground motions", Journal of Structural Engineering, 123(4), pp. 397-404 (1997).

5. Alavi, B. and Krawinkler, H., Effects of Near-Fault Ground Motions on Frame Structures, John A. Blume Earthquake Engineering Center Stanford (2001).

6. Menun, C. and Fu, Q. "An analytical model for near-fault ground motions and the response of SDOF systems", Proceedings of 7th US National Conference on Earthquake Engineering, Boston, Massachusetts, pp. 21-25 (2002).

7. Makris, N. and Black, C.J. Dimensional Analysis of Inelastic Structures Subjected to Near Fault Ground Motions, Earthquake Engineering Research Center, University of California (2003).

8. Akkar, S., Yazgan, U., and Gülkan, P. "Drift estimates in frame buildings subjected to near-fault ground motions", Journal of Structural Engineering, 131(7), pp. 1014-1024 (2005).

9. Luco, N. and Cornell, C.A. "Structure-specific scalar intensity measures for near-source and ordinary earthquake ground motions", Earthquake Spectra, 23(2), pp. 357-392 (2007).

10. Xie, L., Xu, L., and Adrian, R.M. "Representation of near-fault pulse-type ground motions", Earthquake Engineering and Engineering Vibration, 4(2), pp. 191199 (2005).

11. Zhai, C., Li, S., Xie, L., and Sun, Y. "Study on inelastic displacement ratio spectra for near-fault pulsetype ground motions", Earthquake Engineering and Engineering Vibration, 6(4), pp. 351-355 (2007).

12. Ribakov, Y. "Reduction of structural response to nearfault earthquakes by seismic isolation columns and variable friction dampers", Earthquake Engineering and Engineering Vibration, 9(1), pp. 113-122 (2010).

13. Yaghmaei-Sabegh, S. "Detection of pulse-like ground motions based on continues wavelet transform", Journal of Seismology, 14(4), pp. 715-726 (2010).

14. Alonso-Rodríguez, A. and Miranda, E. "Assessment of building behavior under near-fault pulse-like ground motions through simplified models", Soil Dynamics and Earthquake Engineering, 79, pp. 47-58 (2015). 
15. Zhao, W.S. and Chen, W.Z. "Effect of near-fault ground motions with long-period pulses on the tunnel", Journal of Vibroengineering, 17(2), pp. 841-858 (2015).

16. Alhan, C. and Öncü-Davas, S. "Performance limits of seismically isolated buildings under near-field earthquakes", Engineering Structures, 116, pp. 83-94 (2016).

17. Alhan, C., Gazi, H., and Kurtuluş, H. "Significance of stiffening of high damping rubber bearings on the response of base-isolated buildings under nearfault earthquakes", Mechanical Systems and Signal Processing, 79, pp. 297-313 (2016).

18. Chen, Z., Chen, W., Li, Y., and Yuan, Y. "Shaking table test of a multi-story subway station under pulselike ground motions", Soil Dynamics and Earthquake Engineering, 82, pp. 111-122 (2016).

19. Yazdani, Y. and Alembagheri, M. "Effects of base and lift joints on the dynamic response of concrete gravity dams to pulse-like excitations", Journal of Earthquake Engineering, 21(5), pp. 840-860 (2017).

20. Zhao, G.C., Xu, L., and Xie, L. "Study on lowfrequency characterizations of pulse-type ground motions through multi-resolution analysis", Journal of Earthquake Engineering, 20(6), pp. 1011-1033 (2016).

21. Baker, J.W. "Quantitative classification of near-fault ground motions using wavelet analysis", Bulletin of the Seismological Society of America, 97 (5), pp. 1486-1501 (2007).

22. Mavroeidis, G.P. and Papageorgiou, A.S. "A mathematical representation of near-fault ground motions", Bulletin of the Seismological Society of America, 93(3), pp. 1099-1131 (2003).

23. Hoseini-Vaez, S.R., Sharbatdar, M.K., GhodratiAmiri, G., Naderpour, H., and Kheyroddin, A. "Dominant pulse simulation of near fault ground motions", Earthquake Engineering and Engineering Vibration, 12(2), pp. 267-278 (2013).

24. Mimoglou, P., Psycharis, I.N., and Taflampas, I.M. "Determination of the parameters of the directivity pulse embedded in near-fault ground motions and its effect on structural response", In Computational Methods in Earthquake Engineering, pp. 27-48 (2017).

25. Kaveh, A., Hoseini Vaez, S.R., and Hosseini, P. "MATLAB code for an enhanced vibrating particles system algorithm", International Journal of Optimization in Civil Engineering, 8(3), pp. 401-414 (2018).

26. Kaveh, A., Hoseini Vaez, S.R., and Hosseini, P. "Modified dolphin monitoring operator for weight optimization of frame structures", Periodica Polytechnica Civil Engineering, 61(4), pp. 770-779 (2017).

27. Hoseini Vaez, S.R. and Sarvdalir, S. "Reliabilitybased optimization of one-bay 2-D steel frame", KSCE Journal of Civil Engineering, 22(7), pp. 2433-2440 (2018).
28. Kaveh, A., Hoseini Vaez, S.R., and Hosseini, P. "Enhanced vibrating particles system algorithm for damage identification of truss structures", Scientia Iranica, 26(1), pp. 246-256 (2019).

29. Hoseini Vaez, S.R. and Fallah, N. "Damage detection of thin plates using GA-PSO algorithm based on modal data", Arabian Journal for Science and Engineering, 42(3), pp. 1251-1263 (2017).

30. Kaveh, A., Hoseini Vaez, S.R., Hosseini, P., and Fallah, N. "Detection of damage in truss structures using simplified dolphin echolocation algorithm based on modal data", Smart Structures and Systems, 18(5), pp. 983-1004 (2016).

31. Shi, Y. and Eberhart, R. "A modified particle swarm optimizer", Proceedings of the 1998 IEEE International Conference on Evolutionary Computation, pp. 69-73 (1998).

32. Eberhart, R. and Kennedy, J. "A new optimizer using particle swarm theory", Proceedings of the Sixth International Symposium on Micro Machine and Human Science Nagoya, Japan, pp. 39-43 (1995).

33. Kennedy, J. and Eberhart, R. "Particle swarm optimization", Proceedings of the IEEE International Conference on Neural Networks Piscataway, pp. 19421948 (1995).

34. Bazaraa, M.S., Sherali, H.D., and Shetty, C.M. "Nonlinear programming: Theory and algorithms", John Wiley \& Sons, Canada, USA (2013).

35. Coello, C.A.C. "Theoretical and numerical constrainthandling techniques used with evolutionary algorithms: A survey of the state of the art", Computer Methods in Applied Mechanics and Engineering, 191(11), pp. 1245-1287 (2002).

36. McFadden, P.D., Cook, J.G., and Forster, L.M. "Decomposition of gear vibration signals by the generalised S transform", Mechanical Systems and Signal Processing, 13(5), pp. 691-707 (1999).

37. Trifunac, M.D. "Energy of strong motion at earthquake source", Soil Dynamics and Earthquake Engineering, 28(1), pp. 1-6 (2008).

38. Todorovska, M.I., Meidani, H., and Trifunac, M.D. "Wavelet approximation of earthquake strong ground motion-goodness of fit for a database in terms of predicting nonlinear structural response", Soil Dynamics and Earthquake Engineering, 29(4), pp. 742-751 (2009).

\section{Appendix A}

In Figure A.1, the actual record and extracted strong velocity pulse from 91 pulse-like records of Table 2 are shown. The complete fitting of the simulated pulse 


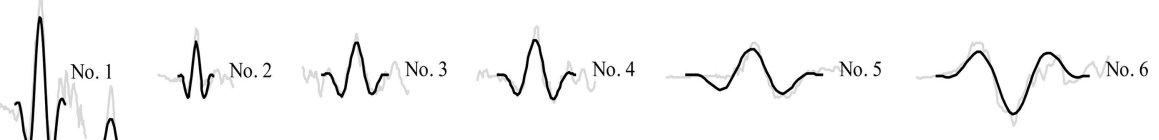

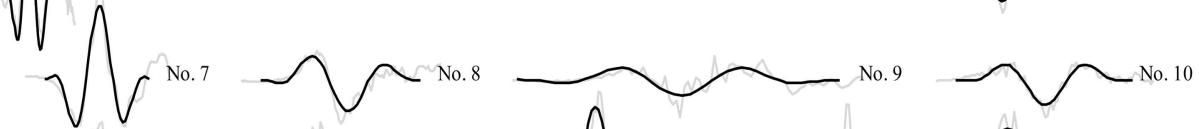

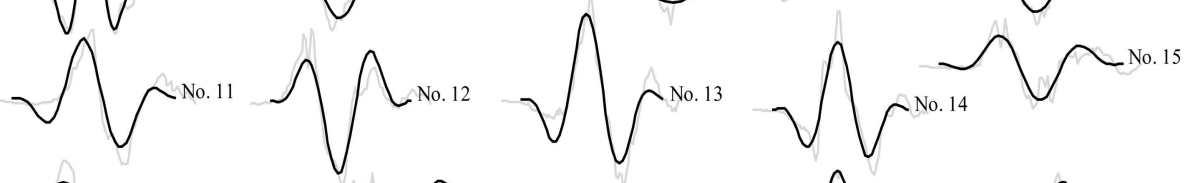

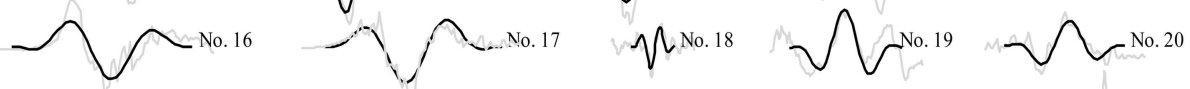

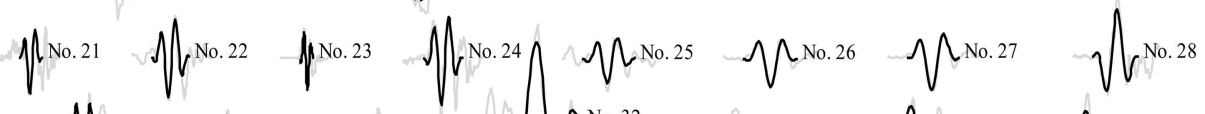

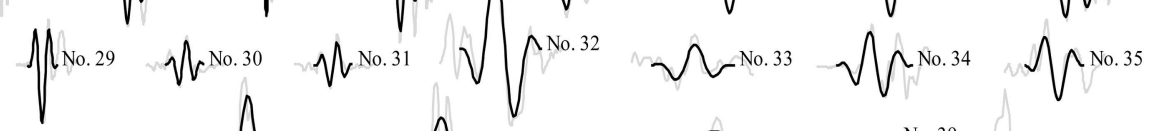

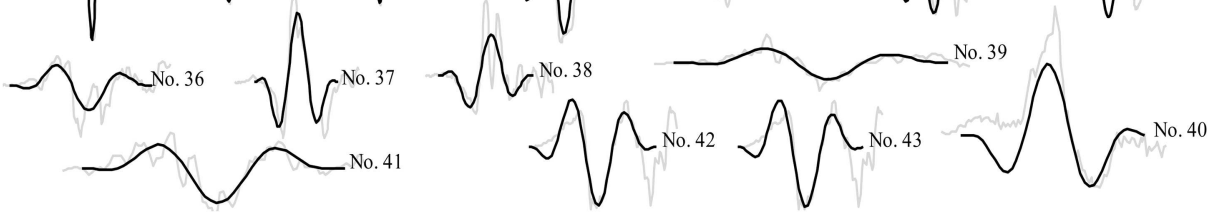

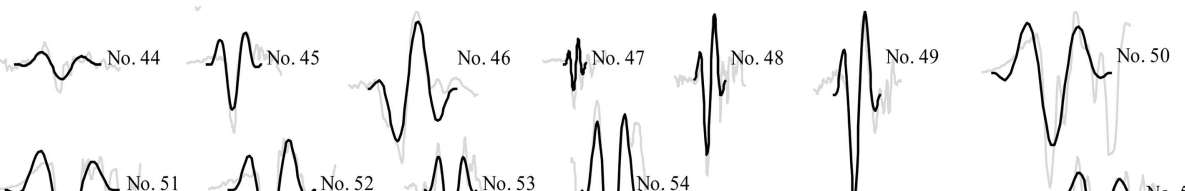

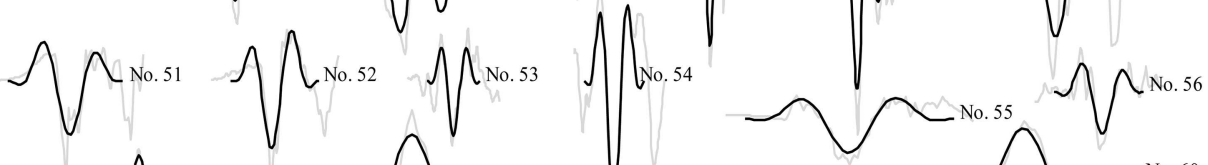
$W^{\text {No.57 }} \sim^{\text {No.58 }} \sim^{\text {No.59 }}$
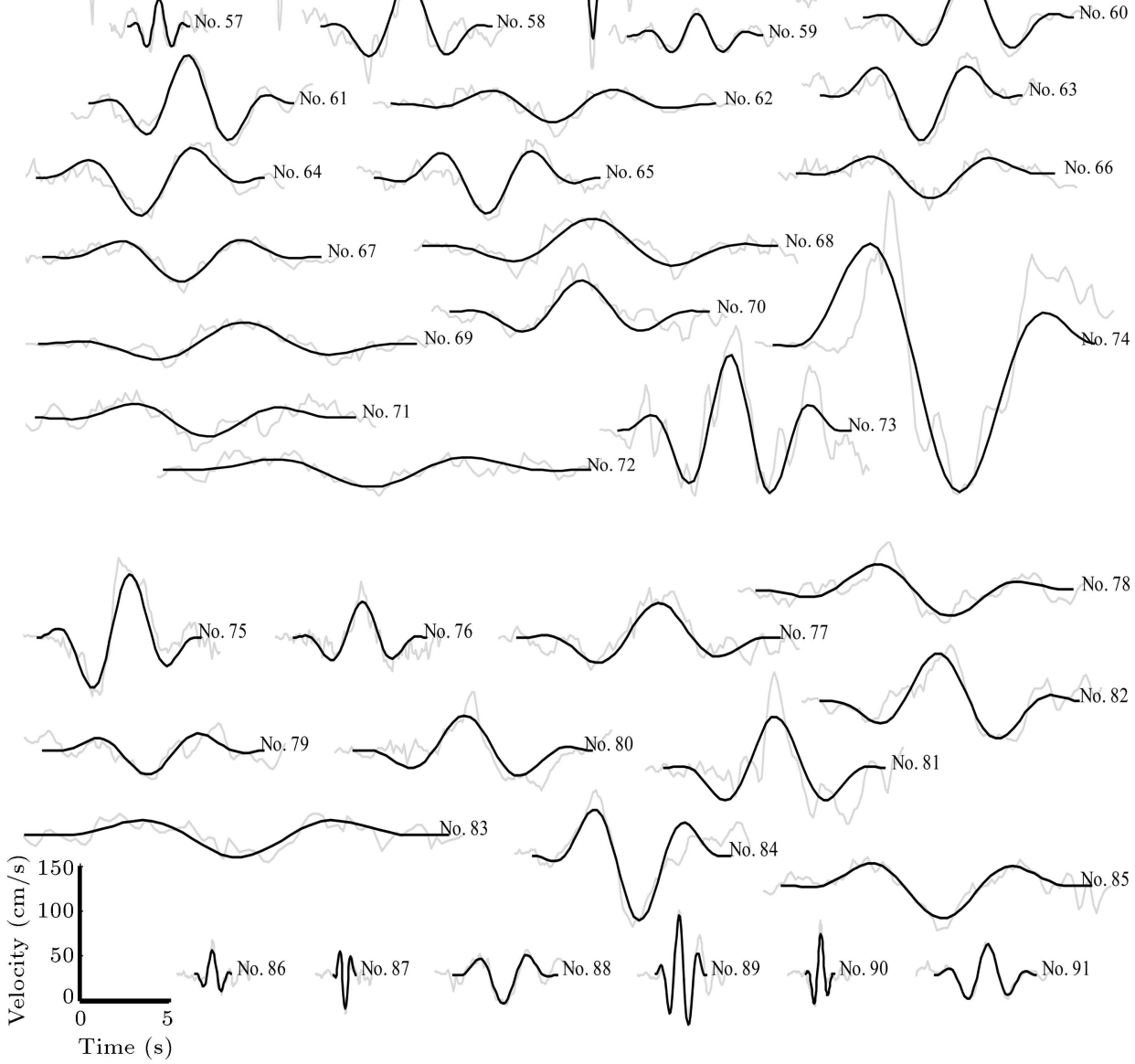

Figure A.1. Extracted strong velocity pulse and the original records of Table 2. 
and the actual record is obtained by substituting the parameters of Table 4 in the analytical model of Hoseini Vaez et al. [23].

\section{Biographies}

Seyed Rohollah Hoseini Vaez is currently an Associate Professor at the University of Qom. He teaches courses on the finite element methods, structural optimization, advanced reinforced concrete structures, and earthquake engineering. His research interests include damage detection, finite element method, optimization algorithms, and near-fault ground motions.

Zahra Minaei graduated with an MSc degree in Civil Engineering, Structural Engineering, from Arak University, Arak, Iran in 2013, where she also received her BSc in 2010. She is currently a PhD candidate in Structural Engineering, at the University of Qom, Qom, Iran. Her research interests include seismic analysis, optimization methods, and fuzzy clustering approach. 